\title{
Zur Erwerbung der Bremer Papyrussammlung und des Apollonios-Archivs
}

\author{
Holger Essler und Maria Hermes-Wladarsch ${ }^{* 1}$
}

\begin{abstract}
In the past, there have been contradictory statements about the acquisition of the Bremer Papyri, particularly in respect of their relationship to the "Deutsches Papyruskartell". The article reconstructs in detail the history of the Bremen collection and the beginning of the Gießen collection by publishing the relevant documents held by Deutsches Archäologisches Insitut in Cairo and the state and university library Bremen.
\end{abstract}

\footnotetext{
* Corresponding authors: Holger Essler, Universität Würzburg, Institut für Klassische Philologie I, Residenzplatz 2 (Südflügel), D-97070, <holger.essler@uni-wuerzburg.de>; Maria Hermes-Wladarsch, Staats- und Universitätsbibliothek Bremen, Dezernat Digitale Dienste: Digitale Bibliothek, Postfach 3301 60, D-28331 Bremen, <hermes@suub.unibremen.de>
}

${ }^{1}$ Der Aufsatz vereint zwei Abschnitte, die ursprünglich jeweils von einem der beiden Autoren geschrieben wurden. Nach der Zusammenfügung haben wir aber beide den Text erneut überarbeitet. Die Abschriften der Briefe des Deutschen Archäologischen Instituts Kairo und der Staatsbibliothek Berlin sowie der Teil des Aufsatzes zur Erwerbung der Papyri stammen von H. Essler, die Abschriften der Briefe der Staats- und Universitätsbibliothek Bremen und der Teil des Aufsatzes zum Schicksal der Papyri in Bremen stammen von M. Hermes. Für die überaus freundliche Betreuung und archivalische Bearbeitung der Archivalien des DAI Kairo danken wir A. Huth, für die Mitteilung von Wilckens Brief an Steindorff A. Cappel ganz herzlich. Dank schulden wir ferner der Staatsbibliothek Berlin, der SuUB Bremen, dem Ägyptischen Museum Georg Steindorff Leipzig (ÄMUL) und dem DAI Kairo für die Erlaubnis, die Stücke hier zu publizieren. Die Rechte an den Archivalien liegen bei der besitzenden Institution. Die Stücke des DAI Kairo sind nach den derzeit aktuellen Signaturen des Archivs zitiert, die wegen der noch laufenden Erfassung mittelfristig in ein neues Signatursystem überführt werden. Viele der Briefe Borchardts, vor allem die Durchschläge der ersten Zeit, weisen starke Papierschäden auf und sind nur schwer lesbar.

Die Transkriptionen folgen in Orthographie und Zeichensetzung dem Original, sind jedoch fortlaufend gedruckt ohne Rücksicht auf die ursprüngliche Zeilenaufteilung. Der Seitenwechsel im Original ist durch Senkrechte mit hochgestellter Seitenzahl gekennzeichnet ( $I^{2}$ etc.). Die Kennzeichnung von Lücken, Zusätzen und Streichungen folgt dem Leidener Klammersystem (U. Wilcken, Das Leydener Klammersystem, APF 10/1932, S. 210f.), die Hervorhebungen sind, soweit nicht anders vermerkt, die des Originals. 
Keywords: Deutsches Papyruskartell, acquisition, papyrus collection Bremen, papyrus collection Gießen, Ludwig Borchardt

DOI 10.1515/apf-2015-0038

Bisher sind über die Erwerbung der Bremer Sammlung zwei widersprüchliche Aussagen veröffentlicht worden: U. Wilcken, der Herausgeber des Editionsbandes der Bremer Papyri, sprach von einer Erwerbung der Papyri durch das Deutsche Papyruskartell. ${ }^{2}$ Hingegen stellte T. Elsmann anhand der in Bremen aufbewahrten Unterlagen zur Erwerbung der Sammlung fest, daß der Ankauf vor der Kartellgründung lag. ${ }^{3}$ Dazu paßt, daß Bremen bei der Entstehung des Deutschen Papyruskartells keine Rolle spielte und insbesondere bei der Unterzeichnung der Satzungen ab November 1902 nicht unter den Gründungsmitgliedern des Kartells firmierte. ${ }^{4}$ Aufgrund des hier neu edierten Archivmaterials des DAI Kairo und der Staats- und Universitätsbibliothek Bremen läßt sich die Erwerbung der Papyri nahezu auf den Tag genau nachzeichnen. Daraus ergibt sich, daß der eigentliche spiritus rector der Ankäufe des Apollonios-Archivs - sowohl für den Bremer als auch für den Giessener Teil - Ludwig Borchardt war. ${ }^{5} \mathrm{Da}$ Borchardt bei der Erwerbung der Bremer Papyri bereits vieles vorwegnahm oder neu einrichtete, was dann im Geschäftsbetrieb des Papyruskartells üblich wurde, mag man durchaus von einer Keimzelle dieser Institution sprechen.

Erworben wurden die Bremer Papyri im Februar 1902 vom Bremer Kaufmann Hermann Melchers auf einer Reise in Ägypten. Anzeichen über

${ }^{2}$ U. Wilcken, Die Bremer Papyri (SBAW 1936,2, Berlin 1936). Zu Ulrich Wilcken (*18. Dezember 1862 in Stettin; $†$ 10. Dezember 1944 in Baden-Baden) vgl. G. Poethke, ,Ulrich Wilcken (1862-1944)', in: M. Capasso (ed.), Hermae, Pisa 2007, S. 81-96, L. Wenger, ,Ulrich Wilcken †', Zeitschrift der Savigny-Stiftung für Rechtsgeschichte. Romanistische Abteilung 65 (1947), S. XI-XX. Sein Nachlass muß größtenteils als verloren gelten, vgl. G. Audring (Hg.), Ulrich Wilcken. Briefe an Eduard Meyer, Konstanz 1994, S. 12.

${ }^{3}$ T. Elsmann: Die ,Bremer Papyri ${ }^{-}$- Zur Geschichte einer Sammlung, in: T. Schmitt, M. Böhme (Hg.): Welt aus Schnipseln. Papyrus-Texte aus dem Alten Ägypten, Bremen 2007, S. 53-56, hier S. 55.

${ }^{4}$ O. Primavesi: Zur Geschichte des Deutschen Papyruskartells, ZPE 114, 1996, S. 173-187, hier S. 179.

${ }^{5} \mathrm{Zu}$ L. Borchardts Tätigkeit für das Kartell vgl. vor allem S. Voss, Die Geschichte der Abteilung Kairo des DAI im Spannungsfeld deutscher politischer Interessen, Bd. 1, 1881 -1929 (Rahden/Westf. 2013), S. 82-87. 
frühere Bemühungen der wissenschaftlichen Bibliothek Bremens, Papyri anzukaufen oder mit Melchers in Kontakt zu treten, gibt es nicht. Um 1900 richtete sich die Erwerbungspolitik der wissenschaftlichen Bibliothek (die zudem erst seit 1863 mit Johann Georg Kohl einen ersten hauptamtlichen Leiter hatte) nicht auf solcherlei „Realien“, deren Sammlung man Privatleuten überließ. Die Schenkung der Bremer Papyri an die Bibliothek ist daher im Zusammenhang des hanseatischen Mäzenatentums $\mathrm{zu}$ verorten, das in der Freien Hansestadt Bremen die Kunst- und Kulturinstitutionen umfangreich unterstützte.

\section{Wilckens Aufruf in Straßburg und die Initiativen Wilhelm Crönerts und Ernst Kornemanns}

Ausgangspunkt für die im Folgenden nachgezeichnete Entwicklung scheint Ulrich Wilckens Vortrag „Der heutige Stand der Papyrusforschung“ vor der „46. Versammlung deutscher Philologen und Schulmänner“ in Straßburg im Elsaß am 2. Oktober 1901 gewesen zu sein. Er ruft darin zur weiteren Gründung von Sammlungen in Deutschland und Spenden zum Erwerb von Papyri auf: ${ }^{6}$ „Es ist daher ein erstrebenswertes Ziel, daß schließlich jede Universität ihre Papyrussammlung habe. Es ist aber auch ein realisierbares Ziel, denn Papyri giebt es über und unter der Erde genug, um noch viele Sammlungen zu bilden. Es bedarf nur der freigebigen Hand, die die Mittel spendet, und der geschickten und glücklichen Vermittler.“ Der Tagungsbericht meldet dazu: ${ }^{7}$ „Der Vortrag fand bei den Versammelten die größte Aufmerksamkeit und rauschenden Beifall, besonders auch aus dem Grunde, weil die Universitäts- und Landesbibliothek [Straßburg, d. Verf.] seit kurzem im Besitz einer eigenen Papyrussammlung ist, die durch das liberale Entgegenkommen des Fürsten Statthalters zum teilweisen Ersatz für die 1870 vernichteten handschriftlichen Schätze angekauft worden ist." Ende des Monats gab Wilhelm Crönert in der in München erscheinenden Allgemeinen Zeitung einen Überblick über die neueren Papyruserwerbungen und plädierte für eine dezentrale Erwerbung von Papyri in Deutschland, die möglichst viele

\footnotetext{
${ }^{6}$ Abgedruckt in NJA 4, 1901, S. 677-691, das folgende Zitat auf S. 680.

${ }^{7}$ Verhandlungen der sechsundvierzigsten Versammlung deutscher Philologen und Schulmänner in Straßburg (Elsaß) vom 1. bis 4. Oktober 1901, Leipzig 1902, S. 37.
} 
private Spender miteinbeziehen sollte. ${ }^{8}$ Er erwähnt auch zwei unterschiedliche Gruppen, von denen die erste einen unitarischen Standpunkt vertritt und alle Papyri in Berlin zentralisieren möchte, während die zweite das Heil in der Zersplitterung sieht, weil dadurch mehr Interesse geweckt, Bearbeiter und Spender gewonnen werden könnten. Er schließt sich wie Wilcken dem föderalen Lager an. In dieselbe Richtung geht auch Richard Reitzenstein, einer der Verantwortlichen für die Straßburger Ankäufe, in seinem Artikel „Deutsche Papyrus-Sammlungen“, der am 11.11.1901 in der Allgemeinen Zeitung erschien. ${ }^{9}$

Crönert scheint auch gleich erste Schritte zur Vorbereitung einer deutschen Grabungsmission getan zu haben, um an die großen Erfolge der Engländer anzuknüpfen. Er versuchte einerseits, Geldgeber in Bremen zu gewinnen, und wandte sich auf Anregung von R. Reitzenstein (Straßburg) gleichzeitig an Ludwig Borchardt, den wissenschaftlichen Sachverständigen beim Kaiserlichen Deutschen General-Consulat für Ägypten mit der Bitte um Unterstützung für ein mögliches Grabungsunternehmen. Borchardt war bereits seit einiger Zeit mit der Erwerbung von Papyri für die Straßburger Sammlung befaßt. ${ }^{10}$ Crönerts Briefe und Durchschläge von Borchardts Antwortschreiben haben sich im Archiv des DAI Kairo erhalten. ${ }^{11}$

${ }^{8}$ W. Crönert: Papyrusneuigkeiten, Beilage zur Allgemeinen Zeitung Nr. 246, 25.10. 1901, S. 1-5 (http://daten.digitale-sammlungen.de/bsb00085657/image_175); das Plädoyer auf S. 5.

${ }^{9}$ R. Reitzenstein, Deutsche Papyrus-Sammlungen, Beilage zur Allgemeinen Zeitung Nr. 259, 11.11.1901, S. 1-2 (http://daten.digitale-sammlungen.de/bsb00085657/image_ 279).

${ }^{10} \mathrm{Vgl}$. dazu etwa R. Reitzenstein, Zwei religionsgeschichtliche Fragen nach ungedruckten griechischen Texten der Strassburger Bibliothek (Straßburg, 1901), S. V sowie L. Borchardt, Bericht über die Thätigkeit des dem Generalconsulate für Aegypten attachirten wissenschaftlichen Sachverständigen Dr. Ludwig Borchardt in der Zeit vom October 1899 bis Juli 1900 (SPAW 5, 1901, Berlin 1901), S. 1 [106].

${ }^{11}$ Der Verbleib von Crönerts Briefnachlaß ist unbekannt. Wenigstens in den 1980er Jahren befanden sich die Briefe nicht mehr in seinem Haus in Schwarzwald. Für die Informationen über seine Nachforschungen zum Nachlaß Crönerts danken wir T. Dorandi ganz herzlich. Die Überführung seiner Bibliothek und Münzsammlung nach Göttingen beschreibt M. Gigante, Atakta. XXII. Per un profilo di Wilhelm Crönert, CErc. 16, 1986, S. 94-99 (Ndr. M. Gigante, Atakta, Napoli 1993, S. 39-48). Beispiele und Typologie von Crönerts Anmerkungen in seinen Büchern bei V. Garulli, Il laboratorio loboniano di Wilhelm Crönert, Eikasmos 16, 2005, S. 487-498. 


\section{Brief Crönert, Bonn, an Borchardt, Kairo, 8.12.1901}

Handschr. Original, DAIK Altakten, B XXIXa Korrespondenz Ankäufe „Kleinere deutsche Sammlungen“ 1899-1912. Darauf der Vermerk „zu 642/02“.

Bonn, Cassiusgraben 2 den 8 XII 1901

Sehr geehrter Herr,

falls die Umstände sehr günstig sind, werde ich nächstens in Bremen einige Mittel für eine deutsche Papyrusgrabung in Ägypten erhalten. Mein Plan, falls die Gelder sich auftreiben lasen, ist folgender:

im Februar des n. J. nach Ägypten zu reisen, um dort die Stätte aufzusuchen, wo ich am besten den Spaten ansetze, und dann um in Kairo einiges aus dem Papyrushandel zu gewinnen. Im Herbst nächsten Jahres soll dann die Hauptarbeit ansetzen (sic). Die Stadt, von der ich die Mittel erwarte, ist Bremen. Am 15. Januar werde ich dort in der vereinigten Geographischen und Literarischen Gesellschaft einen Vortrag über die deutsche Papyrusgrabung in Ägypten halten. Dann muß sich alles ent ${ }^{2}$ scheiden.

Nun bitte ich Sie um die Güte, mir freundlichst mitzuteilen, daß Sie mich, der ich noch nicht in Ägypten war, nicht ohne Beistand und Rat lassen werden. Wenn es angeht, so schreiben Sie mir dies bitte in einem amtlichen, mit Siegel versehenen Schreiben, denn ich werde Ihre Antwort den Bremer Herren vorlegen müssen. Augenblicklich sind, wie Sie wissen, die politischen Verhältnisse in Ägypten noch günstig, das muß notwendig ausgenützt werden. Sie wissen ferner, daß es bis jetzt noch keine erfolgreiche deutsche Papyrusgrabung in Ägypten gegeben hat, während die Engländer alljährlich mit reichen Schätzen ${ }^{\beta}$ ihre Grabungsstätten verlassen. Da ist es denn nicht zum wenigsten eine nationale Aufgabe und eine nationale Pflicht, daß wir Deutschen, die wir für die Erklärung und wissenschaftliche Verwertung die besten Kräfte stellen, nun auch in der Gewinnung nicht zurückbleiben.

Indem ich Sie bitte, nachdem Sie der Straßburger Sammlung so wichtige Dienste geleistet haben - Herr Professor Reitzenstein erzählte mir auf dem Straßburger Philologentage viel von Ihrer thatkräftigen Unterstützung - nun auch die Bremer Angelegenheit nicht aus den Augen zu lassen, bin ich $l^{4}$ mit dem Ausdrucke meiner ganz vorzüglichen Hochachtung

Ihr ergebener

Dr. W. Crönert 
Zum Zeitpunkt seiner Antwort hatte Borchardt bereits seit Juli 1900 zehn Ankäufe von Papyri und Ostraka für Straßburg vermittelt. ${ }^{12}$ Darüber hinaus war er seit dem 1. April 1901 von der preußischen Commission zur Erwerbung griechischlitterarischer Papyri aus Egypten beauftragt, für die Königlichen Museen in Berlin literarische Papyri anzukaufen. ${ }^{13}$ Seit Oktober 1901 hatte die Kommission auch Otto Rubensohn als ständigen Vertreter des preußischen Papyrusunternehmens in Kairo, der Ankäufe durchführte und die erste Berliner Papyrusgrabung vorbereitete. ${ }^{14}$ Die Möglichkeit, daß die verschiedenen Bestrebungen von Papyruserwerbungen zu einander und namentlich zu Berlin in Konkurrenz treten könnten, hebt Borchardt in seinem Antwortschreiben selbst hervor. Dessen ungeachtet bestärkt er Crönert in seinem Vorhaben und sichert seine Unterstützung $\mathrm{zu}^{15}$

\section{Brief Borchardt, Cairo, an Crönert, 19.12.1901}

Handschr. Durchschrift, DAIK Altakten, B XXIXa Korrespondenz Ankäufe „Kleinere deutsche Sammlungen“ 1899-1912. Darauf der Vermerk „zu 642/02“.

Cairo 19/12 01

Sehr geehrter Herr Dr.!

J.No. 380

Ihre Absicht, hier in Aegypten ein deutsches Papyrusunternehmen ins Leben zu rufen, kann ich nur mit der größten Freude begrüßen. Ihnen brauche ich wohl nicht erst zu sagen, wie weit bei uns das praktische Interesse an der Papyrusforschung hinter den schönen Erfolgen zurücksteht, die wir auf diesem Gebiete in wissenschaftlicher Beziehung zu verzeichnen haben. Es wäre wirklich nur zu wünschen, daß auch bei

${ }^{12}$ R. Reitzenstein dankt ihm und anderen Ankäufern für Straßburg im Vorwort seiner ersten größeren Edition, datiert auf den 23.6.1901 (R. Reitzenstein, Zwei religionsgeschichtliche Fragen, Strassburg 1901, S. Vf.).

${ }^{13}$ O. Primavesi: Zur Geschichte des Deutschen Papyruskartells, ZPE 114, 1996, S. 173-187, hier S. 175.

${ }^{14}$ H. Essler, F. Reiter: Die Berliner Sammlung im Deutschen Papyruskartell, in: P. Schubert (Hg.), Actes du 26e Congrès international de papyrologie. Genève, 16-21 août 2010, Genève 2012 (Recherches et Rencontres 30), S. 213-220, hier S. 214.

${ }^{15} \mathrm{Zu} \mathrm{L}$. Borchardts weiteren Auseinandersetzungen mit dem „Berliner Lager“ vgl. S. Voss, Die Geschichte der Abteilung Kairo des DAI im Spannungsfeld deutscher politischer Interessen, Bd. 1, 1881-1929 (Rahden/Westf. 2013), S. 161-164. 
uns bald auch für die Erwerbung dieses so hochwichtigen kulturgeschichtlichen Materials Mittel flüssig gemacht würden, damit wir nicht erst auf dem Platze erscheinen, wenn nur noch die von den anderen Nationen übriggelassenen Reste vorhanden sind. $I^{2}$ Es ist zwar, wie Ihnen bekannt sein dürfte, eine groß angelegte Papyrusunternehmung von preußischer Seite aus im Gange, die sich namentlich die Erwerbung litterarischer griechischer Papyri zum Ziele gesteckt hat, aber das hindert in keiner Weise, daß auch von Seiten eines anderen Bundesstaates für diese Forschung, deren Material nicht [a]llzulange mehr zu beschaffen sein dürfte, etwas gethan wird. Bei der verhältnißmäßig eng begrenzten Aufgabe des preußischen Unternehmens, ist eine Beeinträchtigung nicht zu befürchten, im Gegentheil kann ich mir das Nebeneinanderwirken mehrerer deutscher Papyrusunternehmungen nur als vortheilhaft vorstellen.

Sie wünschen nun meinen Rath in dieser Angelegenheit. Da muß ich sie zuerst nach der Art und Größe der Ihnen zur Verfügung stehenden $l^{3}$ Fonds fragen. Bekommen Sie dieselben als einmalige Bewilligung, oder als mehrjährig laufende?

In ersterem Falle würde ich nicht unbedingt zu einer Ausgrabung rathen, bei der Sie nicht auf sicheren Erfolg rechnen können - auch von den Engländern hören Sie immer nur von den erfol[g]reichen Grabungen, die weniger glücklichen werden in den Berichten mei[st] recht kurz besprochen. Sie würden bei einmalig bewilligten Mitteln riskieren, daß Sie dieselben einer resultatlosen Ausgrabung verausgaben. Auch wenn Sie sich in diesem Falle auf Ankäufe beschränken, würde ich Ihnen rathen, von vorne herei[n] den Fonds übertragbar zu machen, damit Sie nicht gezwungen sind, Ihre Ankäufe im Laufe eines Etatsjahres zu erledigen. Es könnte vorkommen, daß Sie in diesem Jahre $\mathrm{I}^{4}$ nichts ordentliches auf dem Markte fänden. Mit dem doch nur kleinen Straßburger Papyrusfonds, für dessen Verausgabung keine bestim(m)te Frist vorgesehen war, konnten in Folge geduldigen Abwartens doch ganz erfreuliche Ankäufe gemacht werden, was sicher nicht gelungen wäre, wenn die Summe innerhalb eines Etatsjahres hätte verausgabt werden müssen.

Haben Sie aber für mehrere Jahre laufende Mittel, so können Sie schon eher an eine Ausgrabung gehen, da Sie dann Zeit und Gelegenheit haben, erst Erfahrungen beim Graben zu sammeln und wenigstens eines Erfolges bei einer Reihe von Grabungen sicher sein dürften.

Die Höhe der erforderlichen Summe richtet sich nach Dauer und Ausdehnung, die Sie Ihrer Grabung geben wollen. Dieselbe genauer zu verl ${ }^{5}$ anschlagen, ist mir ohne nähere Angaben Ihrerseits unmöglich. Nach meinen Erfahrungen würde ich aber die Inangriffnahme einer Ausgrabung mit geringeren Mitteln als $10000 \mathrm{M}$ widerrathen; hierzu 
müßten dann noch etwa $5000 \mathrm{M}$ für Ankäufe kommen, so daß Sie also einen Jahre[s]betrag von 15000 M bräuchten, um mit Erfolg arbeiten zu können. Bei den Ankäufen hätten Sie allerdings die Concurrenz des preußischen Unternehmens zu bestehen, das über beträchtliche größere Mittel verfügt. Aber darüber wird sich schon irgend ein Arrangement treffen lassen, bei dem keiner der beiden Theile zu kurz kommt.

Ihnen sogleich einen geeigneten Platz für Ihre Ausgrabungen zu nennen, bin ich nicht im Stande.

Vor kurzem erst bin ich von einer $I^{6}$ mit dem Herrn vom Berliner Museum unternommenen Rekognoscierung im Fajûm und in Oberägypten zurückgekehrt, auf welcher wir zwei Plätze für die preußische Grabung ermittelt haben; weitere weiß ich aber vor der Hand nicht, jedoch wird dafür schon Rath zu schaffen sein.

Falls Sie selbst nach Aegypten kom(m)en wollen, kann ich Ihnen nicht dringen[d] genug anraten, sich vorher eine ausreichende Kenntnis der ägyptischen arabischen Umgangssprache zu verschaffen, da Sie sonst hier viel Zeit durch Erlernung derselben verlieren. Falls Sie Neugriechisch sprech[en] sollten, wird das Ihnen bei einigen griechischen Händlern hier von Nutzen sein. Über die Ausrüstung Ihrer Expedition $\mathrm{zu}$ sprechen, ist wohl noch später Zeit. Ich stehe Ihnen stets für $I^{7}$ alle Anfragen zur Verfügung.

$\mathrm{Da}$ ich Ihnen hier in Aegypten nach Kräften zur Seite stehen werde, ist selbstverständlich. Sie können natürlich auch von unseren Ausgrabungen einige geschulte Vorarbeiter bekommen, und auch, falls Sie Lust dazu haben, sich den hiesigen Ausgrabungsbetrieb, so lange Sie es für nöthig halten, bei uns an[sehen.] Vom Anfang Juni an [...] wird eine, später werden zwei deutsche Ausgrabungen hier im Gange sein, die Ihnen dazu Gelegenheit bieten.

Mit vorzüglicher Hochachtung

Ihr sehr ergebener

Dr. Borchardt.

Noch vor dem Eintreffen von Borchardts Antwort tat Crönert weitere Schritte. Auf den 20. Dezember 1901 ist seine Denkschrift betreffend eine deutsche Papyrusgrabung auf dem Boden griechisch-römischer Kultur in Ägypten datiert, in der er zu einem nationalen Papyrusunternehmen und Spenden von privater Seite aufruft, damit Deutschland nicht weiter hinter den großartigen Erfolgen der Engländer zurückbleibe. ${ }^{16}$ Sein Vortrag vom

${ }^{16}$ W. Crönert: Denkschrift betreffend eine deutsche Papyrusgrabung auf dem Boden griechisch-römischer Kultur in Ägypten (Bonn 1901). Er erwähnt (S. 28) die augenblicklich günstige Lage für ein solches Unternehmen und sodann die Stelle Borchardts, ,,der den 
15. Januar führte zum einen zur Gründung eines vorläufigen Ausschusses, der ein Bremer Papyrusunternehmen in die Wege leiten sollte, zum anderen war dadurch das Interesse des ebenfalls beim Vortrag anwesenden Bremer Kaufmannes Hermann Melchers $(* 1842$ in Bremen, $\dagger$ 08.07. 1918) geweckt, der im Zuge seiner bald darauf angetretenen Ägyptenreise die Bremer Papyri ankaufen sollte. ${ }^{17}$

In seinem nächsten Schreiben an Borchardt kündigt Crönert Melchers Ankunft an und bittet um entgegenkommende Aufnahme für den möglichen Förderer. Dem Brief lag die gerade erschienene Denkschrift bei. Zur Einschätzung von Crönerts verschiedenen Aktivitäten mag die Bemerkung beitragen, daß er um den Jahreswechsel mehrere Briefe mit Material aus den herkulanischen Papyri an Wilamowitz, seinen Doktorvater, ${ }^{18}$ gesandt hatte und Ende Januar zu seinem zweiten Studienaufenthalt nach Neapel aufbrach. ${ }^{19}$

\section{Brief Crönert, Bonn, an Borchardt, Kairo, vom 25.1.1902}

Handschr. Original, DAIK Altakten, B XXIXa Korrespondenz Ankäufe „Kleinere deutsche Sammlungen“ 1899-1912.

\footnotetext{
Auftrag hat, alle deutschen Forschungen im Nillande auf das Kräftigste zu unterstützen." Die Datierung am Ende des Textes (S. 31) lautet auf Bonn, den 20. Dezember 1901. Es scheinen darin aber einige Angaben aus Borchardts Brief verwendet, etwa über die Kosten einer Ausgrabung. Da an jenem Tag Crönert Borchardts Brief vom 19. Dezember 1901 noch nicht vorgelegen haben kann, wurden diese Angaben vermutlich in den Druckfahnen hinzugefügt.

${ }^{17}$ Hermann Melchers stammte aus einer alten Kaufmannsdynastie. Im Alter von 20 Jahren war er nach Asien gekommen, wo er zwei Jahre später, 1866, in Hongkong die Firma Melchers \& Co. Hongkong gründete. Zum Zeitpunkt der Erwerbung der Bremer Papyri lebte er wieder in der Hansestadt.

${ }^{18}$ Seine am 2.3.1897 verteidigte Dissertation Quaestiones Herculanenses erschien 1898 bei Teubner, Leipzig.

${ }^{19}$ Am 26.12.1901 schickt er Material zu Demetrios Lakon, insbesondere PHerc. 1013, und kündigt am 1.1.1902 „wichtige Bemerkungen“ zu PHerc. 1012, einem weiteren Werk dieses Autors, an. Den wohl auf Wilamowitz' Fürsprache von der Preußischen Akademie der Wissenschaften geförderten Aufenthalt in Neapel mußte er aus familiären Gründen abbrechen, wie er ihm am 2. Februar 1902 mitteilt; vgl. A. Bertini Malgarini, Gli studi Ercolanesi di Wilhelm Crönert nelle lettere a Wilamowitz, in: Atti del XVII Congresso Internazionale di Papirologia, Bd. 1, Napoli 1984, S. 187-195, hier S. $191 \mathrm{f}$.
} 
Bonn, Cassiusgraben 2

den 25 I 1902

Sehr geehrter Herr Doktor.

haben sie vielen Dank für Ihr ausführliches Schreiben. Es war mir äußerst wertvoll.

Ich habe in Bremen am 15. Januar einen Vortrag gehalten, welcher zum Ergebnis hatte, daß sich ein vorläufiger Ausschuß zum Zwecke der Gründung eines Bremischen Papyrusunternehmens bildete. Dem Ausschusse gehören an wissenschaftlichen Größen die Professoren Wilcken, Reizenstein [sic!] und Wiedemann ${ }^{20}$ an.

Vorläufig gehen wir aber noch nicht weiter vor, weil ein sehr vermögend<er> Bremer Kaufmann, Herr Hermann Melchers $\left.\right|^{2}$ eben für zwei Monate nach Ägypten reisen will. Herr Melchers ist der Vorsitzende der Bremer Geographischen Gesellschaft und da ich meinen Vortrag eben vor dieser Gesellschaft gehalten habe, so hat er ihn angehört. Er steht der Sache wohlwollend gegenüber, möchte sich aber, ehe er sich dazu entschließt, mit seiner Gesellschaft an dem Papyrusunternehmen Teil zu nehmen, persönlich in Ägypten über die heutige Lage unterrichten. Dazu bietet ihm eine ägyptische Reise, die er schon längst geplant hat, die beste Gelegenheit.

Ich denke, daß Herr Melchers in etwa 8-14 Tagen in Kairo ankommen wird. $\left.\right|^{3}$ Seien Sie nun so gütig, dem Herrn über alles Auskunft zu geben (vielleicht machen Sie ihn auch mit dem Generalkonsul bekannt) und wenn es Ihre Zeit zuläßt, so führen Sie ihn einmal auf einen Tag nach dem Fajum zu den dort grabenden Deutschen oder Engländern, damit er sich ein Bild von der Arbeit verschaffen kann. Erzählen Sie ihm auch von dem Papyrushandel, bei dem Sie ja soviel Glück hatten, und wenn gerade ein paar schöne Stücke auf dem Markte sind, so bieten Sie sie ihm zum Kauf an. Eine Probe, denke ich, wird Herr Melchers schon gern mit nach Hause $\mathrm{I}^{4}$ bringen. Sie sehen, daß Sie nun einer eben sich entwickelnden Sache einen großen Dienst leisten können. Lassen Sie sich darum die Mühen nicht verdrießen, die Sie etwa haben sollten, das wird Ihnen der Dank zehnmal vergelten.

Aus der beifolgenden Denkschrift ersehen Sie, wie ich dem Laien den Plan verständlich zu machen suche. Sie ist aus erklärlichen Gründen national gehalten; zeigen Sie sie darum keinem Ausländer, z.B. Grenfell-Hunt.

${ }^{20}$ Gemeint ist wohl der Ägyptologe Alfred Wiedemann (18.7.1856-7.12.1936), der seit dem 4.12.1891 außerordentlicher Professor in Bonn war. 
Indem ich Ihnen nocheinmal für Ihre Freundlichkeiten danke, bin ich in alter Hochachtung

Ihr ganz ergebener

Dr. Wilhelm Crönert

Wollen Sie mir bitte alsbald schreiben, wenn Herr M. Sie verlassen hat. Übrigens, wenn man Ihnen 500 M. schickt, können Sie dafür ein paar anständige Papyri kaufen?

Gleichzeitig scheint Crönert sich um weitere Geldgeber für die geplante Bremer Papyrussammlung umgesehen und sich dabei auch an die Göttinger Akademie der Wissenschaften gewandt zu haben. Dies geht aus einer Antwort Wilckens hervor, die auf eine entsprechende Mitteilung Crönerts Bezug nimmt. Dort berichtet Wilcken, daß außer Berlin und Bremen noch drei weitere Städte Papyri erwerben wollen. Vermutlich sind damit Gießen, Straßburg und Leipzig gemeint. ${ }^{21}$ Gleichzeitig mahnt er zu Stillschweigen.

\section{Brief Wilcken, Würzburg, an Crönert, Bonn, 5.2.1902}

Handschr. Durchschlag, Staatsbibliothek zu Berlin - Preußischer Kulturbesitz, Handschriftenabteilung: Nachl. Ulrich Wilcken, Briefbuch Bl. 648.

\section{Lieber Herr Doctor!}

Leider muß ich mich heute wieder auf eine ganz kurze Antwort beschränken, hoffe aber sehr, Ihnen nächstens noch ausführlicher zu schreiben.

Wenn die Göttinger Akademie für Papyruserwerbungen gewonnen werden könnte, so würde ich das gewiß mit Freuden begrüßen. Aber sie soll dann für Göttingen kaufen, nicht für Bremen. Wie sollte sie auch dazu kommen? Halten wir beide auch daran fest, daß in erster Linie solche Erwerbungen für Universitätsstädte erwünscht 【ist】 'sind', in denen sie als Unterrichtsmaterial dienen können und die ganze Professorenschaft zur Mitarbeit anregen können. Trotzdem wünsche ich natürlich auch Bremen den besten Erfolg, aber die Gött(in)g(er) Akade-

${ }^{21}$ Leipzig hatte bis dahin allerdings nur Ostraka angekauft. Es ist auch möglich, daß er bereits an Würzburg dachte, für das er spätestens im April 1902 Mittel zum Ankauf von Papyri zur Verfügung hatte. 
mie wollen wir noch im Hintergrunde lassen, damit sie im $\mathrm{l}^{2}$ geeigneten Moment für Göttingen eintreten kann.

Zur Orientierung will ich Ihnen im Vertrauen mitteilen, daß ich noch außer Bremen 'und außer Berlin ${ }^{22}$ nun 3 Städte 'in Deutschland' sehe, die jetzt an Papyruserwerbungen herangehen wollen. Lassen wir diese 4 doch zunächst mal ihr Glück versuchen. Wenn gar zu viele auf ein Mal losgehen, müssen sie sich notwendig gegenseitig schädigen, und manche werden vielleicht eher zurückgestoßen von weiteren Aufwänden, wenn sie nicht gleich einen sehr guten Erfolg haben. Nach Ihrer Auffassung scheint das Unternehmen in Bremen ja in gutem Zuge zu sein. Ich glaube, es ist das einzig Richtige, die Bremer auf ihre eigene Kraft sich stützen zu lassen, und ihnen nicht Aussicht auf Hilfe von auswärts zu eröffnen.

Mit bestem Gruß in Eile

Ihr UWilcken.

\section{Darunter kleiner:}

Noch eine prinzipielle Bemerkung:

Ich halte es für gut, daß die Städte einstweilen im Stillen vorgehen. ${ }^{23}$

Einer der von Wilcken genannten weiteren Interessenten war von ihm selbst an Borchardt verwiesen worden: Ernst Kornemann, ${ }^{24}$ damals Privatdozent in Gießen, waren von einem privaten Geldgeber insgesamt 2000 Mark zum Aufbau einer Lehrsammlung an der Universität Gießen in Aussicht gestellt worden. Dem Rat Wilckens folgend hatte er Borchardt bereits im Januar 1902 um Vermittlung von Papyrusankäufen gebeten. Er gibt dabei auch der Hoffnung Ausdruck, durch den Erwerb von aufsehenerregenden Stücken weitere Geldgeber und vielleicht die Unterstützung des hessischen Großherzogs zu gewinnen. Er ist bereits über das Preußische Papyrusunternehmen informiert und versucht, die Konkurrenz zu vermeiden.

\footnotetext{
${ }^{22}$ Mit diesem Zusatz am oberen Rand wurde das folgende „diese 4“ unzutreffend, ohne daß jedoch eine Korrektur erfolgte.

${ }^{23}$ Der letzte Teil des Satzes ist schwer zu entziffern. Ich vermute für den Fortgang: „bis sie was gutes haben, nicht vorher in der Oeffentlichkeit ... ."

${ }^{24}$ Geboren am 11.10.1868 in Rosenthal bei Kassel, gestorben am 4.12.1946 in München. Noch vor dem Eintreffen der ersten Ankäufe folgte er einem Ruf auf eine außerordentliche Professur für Alte Geschichte in Tübingen.
} 


\section{Brief Kornemann, Giessen, an Borchardt, Kairo, 9. Januar 1902}

Handschr. Original, DAIK Altakten, B XII Korrespondenz Ankäufe Giessen 1902-1905, 1907, 1910.

Giessen, Frankfurterstr. $58^{\mathrm{II}}$

Sehr geehrter Herr!

9/I 1902

Auf Anraten des Herrn Professor Wilcken gestatte ich mir diese Zeilen an Sie zu richten.

Im Herbste vorigen Jahres erhielt ich von einem hiesigen Kapitalisten das Versprechen, dass er mir ca $10.000 \mathrm{M}$ zur Erwerbung von Papyri zur Verfügung stellen würde. Da ich damals gerade im Begriff war zu einer Studienreise nach Italien und Nordafrika (Tunis und Algier) aufzubrechen, konnte ich momentan keinen Gebrauch von dem Anerbieten machen. Als ich nun in diesem Winter an die Ausführung des $1^{2}$ Projektes gehen und eine Reise nach Aegypten unternehmen wollte, hat mein Hintermann einen teilweisen Rückzug angetreten, mit Rücksicht auf die augenblickliche schwere wirtschaftliche Depression (er ist Industrieller) sei es ihm unmöglich, sein Versprechen ganz aufrecht zu erhalten. So sind mir nur $2000 \mathrm{M}$. zur Verwendung übrig geblieben. Ich will nun, so schwer es mir 'auch' wird, auf die Reise nach Aegypten verzichten, um alles Geld für Reiseunkosten zu sparen, und frage bei Ihnen an, ob Sie einen Ankauf von Papyri für mich bezw. meinen Hintermann übernehmen würden. Von den 2000 M. kann ich Ihnen, sobald $\mathrm{I}^{\beta}$ ich Ihre zustimmende Antwort in Händen habe, sofort 1000 M. anweisen lassen, während das zweite Tausend ein oder zwei Monate später folgen würde.

Wie steht es eben mit dem Papyri-Markt? Die Schwierigkeiten der Ankäufe 'Ihrerseits' für einen Dritten nicht verkennend, möchte ich 'doch' bemerken, dass die Sammlung der hiesigen Universität zu Lehrzwecken zur Verfügung gestellt werden soll und zwar sowohl den Philologen wie den Historikern, dass also unser Bestreben wäre litterarische und historische Stücke und zwar solche aus den verschiedenen Zeiten zu bekommen.

Erscheint Ihnen der Zeitpunkt zum Kauf für uns eben nicht günstig wie ich höre, ist augenblicklich eine grössere Unternehmung für Berlin $\mathrm{I}^{4} \mathrm{im}$ Gange - so warten wir lieber noch etwas. Gewinnen wir nämlich das eine oder andere interessante Stück, so habe ich begründete Hoffnung, weitere Kapitalisten in Hessen für die Sache gewinnen zu können, vielleicht unseren Grossherzog selbst zu erwärmen. Ich würde 
daher ganz gern auch auf ein oder das andere grössere Stück den grös-

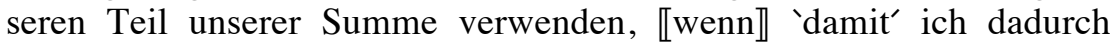
gewissermassen einen Lockvogel zum Heranziehen neuer Geldmänner für meine Sache gewänne.

Doch ich will nicht zu viel Wünsche aussprechen, sondern erst Ihre Antwort abwarten, die ich mir höflichst in Bälde erbitte

Mit Versicherung vorzüglichster

Hochachtung bin ich

Ihr ergebenster

Dr. Ernst Kornemann

Privatdozent der alten Geschichte

an der Universität Giessen.

Borchardt antwortet am 20.1.1902 auch Kornemann entgegenkommend, sagt seine Unterstützung zu, jedoch unter dem Vorbehalt, daß er kein Fachmann für griechische Papyri sei und es sich immer schwierig gestalte, für Fremde zu kaufen. ${ }^{25}$ Kornemann gibt ihm daraufhin freie Hand und kündigt für die Ankäufe die Überweisung von 1000 Mark nach Kairo an. Erneut betont er die Wichtigkeit spektakulärer Ankäufe, um weitere Mittel einzuwerben.

\section{Brief Kornemann, Giessen, an Borchardt, Kairo, 9. Februar 1902}

Handschr. Original, DAIK Altakten, B XII Korrespondenz Ankäufe Giessen 1902-1905, 1907, 1910.

Giessen, Frankfurterstr. 58

Sehr geehrter Herr Borchardt!

9/II 1902

Verbindlichsten Dank für Ihren Brief vom 20. Januar, zu dessen Beantwortung ich leider erst heute komme. Zunächst also auch herzlichen Dank für Ihre Bereitwilligkeit meine Bitte zu erfüllen. Dass es nicht angenehm ist, für Fremde einen solchen Auftrag auszuführen, gebe ich Ihnen unumwunden zu. Das ganze Papyruskaufen scheint mir ein bisschen Lotteriespiel zu sein. Es ist ein gut Stück Risiko dabei. Selbst-

${ }^{25}$ Die handschr. Durchschrift des Briefes aus Abusir, DAIK Altakten, B XII Korrespondenz Ankäufe Giessen 1902-1905, 1907, 1910, ist recht zerstört, doch läßt sich dieser Inhalt noch erkennen. 
verständlich werde ich Ihnen $I^{2}$ nie einen Vorwurf machen, wenn auch ein oder das andere Stück nicht nach meinem Wunsche ist. Denn ich ersehe aus Ihrem Brief, dass Sie Ihr Bestes thun wollen, und das genügt mir und mei[nen] Hintermännern.

Um dann vor allem ein Mi[ss]verständnis aus dem Wege zu räu$\mathrm{m}$ [en:] ich bestehe absolut nicht auf dem alleinigen Ankauf von litterarischen Stücken. Als Historiker sind mir Urkunden und sonstige historische Stücke geradeso willkommen. [Ich] bat nur um Erwerb von einigen grösseren Stücken, nicht nur F[rag]menten und Fragmentchen. [Ich] hätte gern, wie ich Ihnen schon schrieb, ein paar Lockvögel, da ich vorhabe ev(entuell) unseren Grossherzog $\left.\right|^{3}$ für die Sache zu interessieren. Weiter wäre es mir sehr lieb, wenn ich mit Rücksicht auf die Lehrzwecke, denen meine Sammlung dienen soll, Stücke aus den verschiedensten Zeiten (ptolemäische, römische, byzantinische 【)】, vielleicht auch ein oder das andere arabische und vorptolemäische) haben könnte. Was Ostraka betrifft, die mir natürlich auch sehr willkommen sind, so möchte ich anfragen, ob bei denen nicht Fälschungen vorkommen. In diesem Falle bitte ich natürlich um Vorsicht.

Und nun noch eins! Ist eben am Ende des Winters die Zeit zum Erwerben von Papyri nicht ungünstig? Würden Sie vielleicht für geraten halten, die Sommerl ${ }^{4}$ campagne dieses Jahres noch abzuwarten? Auf alle Fälle keine Ueberstürzung! Vielleicht lassen wir auch erst die Hochflut der Berliner Ankäufe vorübergehen. Ich möchte auch nicht nur die Abfälle [der] Berliner kaufen. Doch alles das können Sie aus der Nähe viel besser beurteilen als ich in mein[er] Studierstube. Ich gebe Ihnen in jeder Beziehung vollkommen freie Hand. Die erste Rate von 1000 Mark steht Ihnen jederzeit zur Verfügung; ich bitte nur um eine Mitteilung, wann und in welcher Weise (etwa 【an】 'durch' ein Bankko[nto] in Cairo?) Sie die Summe überwi[esen] haben wollen.

Mit nochmaligem aufrichtigem Dank und dem Ausdruck vorzüglichster Hochachtung bin ich bestens grüssend

Ihr ergebenster

Dr. Ernst Kornemann.

Im Februar bewilligte schließlich die Königlich Sächsische Akademie der Wissenschaften $2000 \mathrm{M}$ für den Erwerb griechischer Papyri, ${ }^{26}$ so daß sich innerhalb weniger Wochen zu Straßburg als neben Berlin auftreten-

${ }^{26}$ Ein Brief G. Steindorffs vom 16.2.1902 an Borchardt, in dem er von diesem Beschluß berichtet und um Vermittlung der Ankäufe bittet, ist erhalten in DAIK Altakten, B XXI Korrespondenz Ankäufe Ägyptologisches Institut der Universität Leipzig 1900-1902, 1905-1909. 
den deutschen Interessenten am Papyrusmarkt noch Bremen, Gießen und Leipzig hinzugesellten.

\section{Berlin und die Konkurrenz}

Inzwischen begann die Berliner Kommission zur Erwerbung griechischlitterarischer Papyri aus Ägypten auf die stetig wachsende Zahl von Aufträgen zum Ankauf von Papyri zu reagieren, die an Borchardt gelangten. Das Vorgehen der Kommission war dabei in Zielen und Mitteln je nach Art der Konkurrenz unterschiedlich. Während man mit den außerpreußischen Institutionen Straßburg und dann auch Gießen und Leipzig eine Einigung anstrebte, suchte man das innerpreußische Unternehmen des jungen Gelehrten Crönert zu unterbinden. Zunächst scheint Hermann Diels, der ebenso wie Crönerts Doktorvater Wilamowitz Mitglied der Berliner Papyruskommission war und mit Crönert in Korrespondenz stand, ihm mitgeteilt zu haben, daß er für sein Vorhaben nicht auf Rubensohns oder Borchardts Unterstützung rechnen dürfe. Borchardt erblickte darin eine unzulässige Einschränkung seiner Tätigkeit und eine Beschneidung seiner Stellung als Reichsbeamter, der alle deutschen wissenschaftlichen Bestrebungen zu fördern habe. Sobald er durch Rubensohn von diesem Schritt der Kommission erfuhr, leitete er Gegenmaßnahmen ein: Noch von der Grabung in Abusir schreibt er am selben Tage (29.1.1902) sowohl an Crönert, um ihn seiner Unterstützung zu versichern, als auch an Erman, dem er in einem längeren Brief seinen Standpunkt in der Frage darlegt und die bisherige Korrespondenz mit Crönert übersendet mit der ausdrücklichen Erlaubnis, diese auch der Berliner Papyruskommission vorzulegen. ${ }^{27}$

\section{Brief Borchardt, Abusir, an Crönert, 29.1.1902}

Handschr. Durchschrift, DAIK Altakten, B XXIXa Korrespondenz Ankäufe „Kleinere deutsche Sammlungen“ 1899-1912. Darauf der Vermerk „zu 642/02".

\footnotetext{
${ }^{27}$ Handschr. Durchschrift dieses Briefes in DAIK Altakten, B XXIXa Korrespondenz Ankäufe „Kleinere deutsche Sammlungen“ 1899-1912.
} 


\section{Sehr geehrter Herr Dr.!}

Wie ich aus einem Schreiben der Generalverwaltung der Königlichen Museen erfahre, hat Herr Geh. Regierungsrath Prof. Diels, Ihnen mitgetheilt, daß ich jetzt wohl keine Zeit finden würde, Sie in dem von Ihnen geplanten Papyrusunternehmen zu unterstützen, oder ähnlich. Ich kenne den Wortlaut des von Herrn Diels an Sie gerichteten Schreibens nicht, möchte aber daraufhin nochmals betonen, was ich Ihnen schon in meinem Briefe vom 19ten Dec. v. J. schrieb, daß ich mich meiner Pflicht Ihnen, wie jedem anderen Deutschen Reichsl ${ }^{2}$ angehörigen hier in Aegypten in wissenschaftlichen Angelegenheiten mit Rath u. That zur Seite zu stehen, nicht entziehen werde. Sie können also auf meine Hilfe rechnen und brauchte Sie die Befürchtung, hier im Lande keine Unterstützung zu finden, von Ihrem Plane nicht abzubringen.

Wenn Sie mich über den Stand der Angelegenheit etwas informieren wollten, würden Sie sehr verpflichten

Ihren hochachtungsvoll ergebenen

Borchardt.

Borchardts Brief an Erman und die Preußische Papyruskommission bringt den Stein ins Rollen und führt schließlich zur Gründung des Deutschen Papyruskartells. Mit diesem Schritt wird die Entwicklung der Angelegenheit auf eine neue Ebene gehoben. In Berlin scheint man sofort nach Erhalt des Schreibens aktiv geworden zu sein. Bereits am 10.2.1902 telegraphiert Richard Schöne, der Vorsitzende der Papyruskommission, an Borchardt und bittet ihn, gegenüber Crönert möglichste Zurückhaltung zu üben und in Bezug auf Straßburg eine Vereinbarung abzuwarten. ${ }^{28}$ Kurz darauf, am 19.2.1902, erklärt sich der preußische Kultusminister mit einer Denkschrift der Berliner Kommission einverstanden.$^{29}$ Sie war von allen

${ }^{28}$ Originalbeleg des Telegramms von Schöne, Berlin, an Borchardt, Cairo Pyramids, 10.2.1902, 2:57 Uhr, in DAIK Altakten, F II Deutsches Papyruskartell. Korrespondenz mit Kgl. Museen zu Berlin 1901-1906: ,erbitte dem bonner Gelehrten gegenueber moeglichste Zurückhaltung und wegen strassburg Verstaendigung abwarten $=$ Schöne $=$.

${ }^{29}$ Eine Abschrift ist abgedruckt als Dokument 1 bei O. Primavesi, Zur Geschichte Des Deutschen Papyruskartells, ZPE 114, 1996, S. 173-187, hier S. 180f. Primavesi datiert das Schreiben auf Januar/Februar 1902. Ein maschinenschriftlicher Entwurf des Schreibens, rechtsspaltig mit handschriftlichen Zusätzen (die bei ihm im Text sind) und eigenhändigen Unterschriften von Ulrich v. Wilamowitz-Moellendorff, Hermann Diels, Adolf Erman und Richard Schöne befindet sich im Politischen Archiv des Auswärtigen Amtes unter der Signatur R 138393. Auf der ersten Seite der handschriftliche Vermerk: „Einverstanden. Der Cultusminister. Studt 19/2 02“. 
Mitgliedern der Kommission unterzeichnet: Richard Schöne, Adolf Erman, Hermann Diels und Ulrich v. Wilamowitz-Moellendorff. Nach einem kurzen Abriss über den Zweck des preußischen Papyrusunternehmens, die bisher bewilligten und in Aussicht gestellten Mittel und bereits erfolgte Ankäufe steht darin vor allem die stetig wachsende Konkurrenz durch andere Aufträge aus Deutschland, die an Borchardt herangetragen werden, im Mittelpunkt. Insgesamt werden vier Konkurrenten aufgeführt: 1) Straßburg, das bereits vor Berlin zu kaufen begonnen hatte, und nun weitere 3000 Mark bereitstelle 2) Kornemann, der 2000 Mark zum Ankauf einer Lehrsammlung für Gießen überwiesen habe, 3) Crönert und 4) Leipzig, das neben den bisher erworbenen Ostraka nun auch den Ankauf von Papyri plane. In dieser Lage hält die Kommission es für geboten, eine Einigung der Konkurrenten herbeizuführen und bis dahin Borchardt anzuweisen, den älteren preußischen Aufträgen die Priorität zu wahren.

Die Angaben über die Entwicklung in Ägypten beruhten auf den Nachrichten, die Rubensohn in einem Bericht an die Kommission mitgeteilt hatte, und sind in einigen Einzelheiten, wie etwa der von Kornemann bereitgestellten Summe, ungenau. Insbesondere aber scheint der von Crönert erwähnte vorläufige Ausschuß zum Zwecke der Gründung eines Bremischen Papyrusunternehmens als Bedrohung empfunden worden zu sein. Im Bericht der Berliner Papyruskommission wird daraus ein privat finanziertes Vorhaben von ähnlichem Zuschnitt wie das preußische. Als Geldgeber wird ein wohlhabender Bonner Privatmann genannt. Es ist unklar, ob hier wegen Crönerts Verbindung zu Bonn irrtümlich Melchers als Bonner Bürger angesehen wurde, oder ob weitere mögliche Förderer bereitstanden. Wenn es sie gab, sind sie nirgends sonst belegt. Bis zu diesem Zeitpunkt lag jedenfalls keine explizite Zustimmung von Melchers zum Kauf von Papyri vor:

3. Dr. Crönert, ein jüngerer Gelehrter in Bonn, hat in Verbindung mit einigen anderen Gelehrten (Professor Wilcken in Würzburg, Reitzenstein in Straßburg und Wiedemann in Bonn) ein dem unsrigen verwandtes Unternehmen zum Zwecke von Ankäufen und Ausgrabungen geplant und soll dazu erhebliche Mittel von einem wohlhabenden Bonner Privatmann erhalten haben.

Daß die ursprüngliche Quelle dieser Angaben Crönerts Mitteilung in seinem Brief an Borchardt vom 25. Januar $1902 \mathrm{zu}$ suchen ist, ergibt sich neben der identischen personellen Zusammensetzung der Kommission aus der Berufung auf den Bericht Rubensohns als Quelle. Rubensohn seiner- 
seits hatte die Nachrichten von Borchardt erhalten. Ein Widerhall dieser Vorbehalte findet sich auch in der Korrespondenz Wilckens, der mehr als einen Monat später auf einen entsprechenden Brief W. Schubarts reagiert und zunächst privat an Heinrich Schäfer schreibt: ${ }^{30}$

\section{Brief Wilcken, Würzburg, an Schäfer, Berlin, 29.3.1902}

Handschr. Durchschlag, Staatsbibliothek zu Berlin - Preußischer Kulturbesitz, Handschriftenabteilung: Nachl. Ulrich Wilcken, Briefbuch, Bl.675.

29.3.02.

\section{Lieber Heinrich!}

Soeben höre ich von Schubart, daß Ihr schon vor einiger Zeit mit Staunen 'aus Aegypten' gehört habt, daß ich mit Reitzenstein und Wiedemann ein Unternehmen leite, das im Wesentlichen dieselben Zwecke verfolgt wie Eure Papyruscommission. Dieses süddeutsche Unternehmen gehe anscheinend von Straßburg aus u.s.w. 'Er wundert sich (mit Recht), daß ich Euch nicht davon geschrieben.'

Ich bin Schubart sehr dankbar, daß er mir dies ganz offen schreibt, und mir Gelegenheit giebt, ein Missverständnis zu beseitigen.

Ich will die ganze Angelegenheit und was damit zusammenhängt ihm darlegen in einem Brief, der ihn nach seiner Rückkehr aus Trebnitz im Museum erwarten soll. Mir ist dieser falsche Verdacht, in dem ich

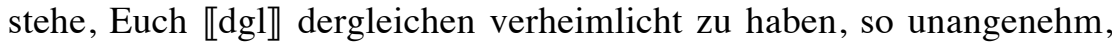
daß ich ihn auch nicht eine Stunde länger auf mir sitzen lassen möchte, und zumal I Ihr jetzt mit Steindorff gewiß viel über solche Dinge geredet, möchte ich Dir schon heute die Erklärung abgeben, daß ein solches „süddeutsches Unternehmen“" zur Zeit gar nicht existiert, wenig-

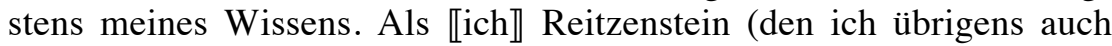
persönlich sehr schätzen gelernt habe) mir im Herbst in Straßburg ein gemeinsames süddeutsches Vorgehen vorschlug, sagte ich ihm, daß das im Princip sehr schön sei, daß ich aber bei meinen persönlichen und früher amtlichen Beziehungen zu Berlin mich nur beteiligen könne, wenn 【je】 es im vollen 'offenen' Einvernehmen mit Eurem Unterneh-

${ }^{30}$ Zur Biographie Wilhelm Schubarts (1873-1960), Wilckens Schüler und damals Direktorialassistent an den Königlichen Museen zu Berlin, vgl. G. Poethke, 'Wilhelm Schubart (1873-1960)', in: M. Capasso (Hg.), Hermae. Scholars and Scholarship in Papyrology, Pisa 2007, S. 193-205, und R. Fikentscher, Liebe, Arbeit, Einsamkeit, Halle [Saale] 2013), S. 437-443. Zu Heinrich Schäfer (1868-1957), der seit 1893 Direktorialassistent war, vgl. A. Wagner, Schäfer, Johann Heinrich, in: Neue Deutsche Biographie, Bd. 22, Berlin 2005, S. $507 f$. 
men, und ohne jede Spitze gegen Berlin gemacht würde (was er denn auch für selbstverständlich hielt).

Weiter ist die Sache noch nicht gediehen. Doch alles weitere will ich an Schubart schreiben. So wie in diesem Falle werde ich es immer als meine Aufgabe betrachten, zu vermitteln und auszugleichen und, wenn nötig, Spitzen gegen Berlin abzubrechen. $\mathrm{Da}$ ich nichts hinter Eurem Rücken treiben will, zeigt wohl am besten, daß, wo immer ich um Rat gefragt werde wegen Papyrusankäufen 'etc.', ich die Leute stets direct an unsern Borchardt verweise. Nein ich hoffe, Ihr habt mir noch ernstlich nichts Schlechtes zugetraut. Ich würde mich freuen, auch von Dir ein Wort darüber zu hören. Mit bestem Gruß Dein

\section{Ulrich}

\section{Der Bremer Ankauf}

Während der schwebenden Verhandlungen und Auseinandersetzungen zwischen Borchardt, der Berliner Papyruskommission und den übrigen deutschen Interessenten war Hermann Melchers nach Ägypten gekommen. Am Montag, dem 10.2.1902 hatte Schöne wie erwähnt an Borchardt telegraphiert und zur Zurückhaltung gegenüber Crönerts Plänen gemahnt. Am Mittwoch, dem 12.2.1902, berichtet Borchardt Melchers von einem größeren Papyrusfund, den der Händler Mohammed Abdallah aus Medinet el Fayum anbot. Insgesamt handelt es sich um sieben Kisten mit dokumentarischen Papyri aus Eschmunen. Kern des Fundes ist das Archiv des Strategen Apollonios. ${ }^{31}$ Melchers zögert zunächst mit dem Ankauf wegen des hohen Preises und der unbekannten Materie, entschließt sich aber tags darauf, die drei bedeutendsten Kisten davon zu erwerben.

\section{Brief Melchers, Kairo, an Borchardt, 13.2.1902. Abbildung 1-4.}

Handschriftl. Original auf Briefkopf der $C^{i e}$ Internationale des grandes Hôtels, DAIK Altakten, B XXIXa Korrespondenz Ankäufe „Kleinere deutsche Sammlungen “ 1899-1912.

Sehr geehrter Herr Professor,

${ }^{31}$ TM Archive Nr. 19. O. Montevecchi, La papirologia, 1988, S. 253 Nr. 34. 
Im Besitz Ihrer freundl(ichen) Zeilen, erlaube ich mir Ihnen zunächst meinen Dank auszusprechen für Ihre Bemühungen, sowie für Ihren Besuch, den ich nach meiner Rückkehr von Luxor \& Assuan nur zu gern an der Stätte Ihrer Thätigkeit erwidern werde.

Was den Ankauf von Papyri anbelangt, so werde ich gern Ihrer Empfehlung folgen, N.o 1-3 zum Preise von 25,- zu kaufen, um unserm $1^{2}$ Bremer Museum ein Geschenk damit zu machen. Wenn ich den Ankauf gestern nicht sofort perfect machte, so geschah es weil ich mich als vollständiger Laie dieser geriebenen ${ }^{32}$ Materie gegenüber etwas unsicher fühlte und weil ich im Hinblick auf Sie - der Autorität - den Kauf gern ganz durch Sie abgeschlossen sähe. Ich hoffe, geehrter Herr Doctor, daß Sie mich nicht für zu unbescheiden halten wenn ich Sie nochmals hierum bitte, indem mich eine kleine Beschreibung der Papyri, die ich dem Museum mit geben könnte, mich zu höchstem Dank verpflichten würde. Dem Abdallah bin ich zwar bereit die genannte Commission von $£ 3$,- zu zahlen, und $\mathrm{I}^{3}$ würde ich die Summe von $£$ 28,- schon hier beifügen, wenn mir dies - da ich die Sicherheit der Postverhältnisse nicht genügend kenne, nicht etwas gewagt erschiene.

Vielleicht können Sie den Abschluß mit Abdallah perfect machen, mit der Bedingung daß ich Ihnen das Geld bei meinem Besuch überbringe, womit Sie mich weiter verpflichten würden.

Nach meiner Rückkehr werde ich mir erlauben mich bei Ihnen anzumelden \& indem mein Pflegesohn \& die übrigen Herren Ihre Grüße bestens erwiedern, bin ich mit freundlicher Empfehlung

Ihr ganz ergebener

Hermann Melchers

aus Bremen $1^{4}$

Da ich einige mich bei Ihnen einführende Zeilen verlegt habe, gestatte ich mir den einst mir von Professor Schauinsland ${ }^{33}$ geschriebenen Brief beizulegen, den ich bei Ihnen ja wieder in Empfang nehmen könnte. d.O.

Eine Woche später meldet Borchardt den erfolgten Ankauf der Stücke. Er legt den Kaufpreis einstweilen für Melchers aus und kann noch sechs weitere Stücke als Zugabe für den veranschlagten Preis bekommen. Seine erste grobe Beschreibung der Stücke als teilweise amtliche Dokumente

${ }^{32}$ Das Wort ist unsicher gelesen. Vermutlich ist „,gerieben“ im Sinne von „Gerissenheit erfordernd" gemeint.

${ }^{33}$ Hugo Schauinsland (1857-1937), Direktor des Bremer Übersee-Museums von 1896 1933. Er wird später von Melchers einige Papyri für das Museum erhalten, vgl. Anm. 49. 
nennt bereits den Strategen Apollonios, die Hauptperson des Archivs. Gleichzeitig leitet er die nächsten Schritte für die konservatorische und die wissenschaftliche Bearbeitung ein, indem er Melchers jeweils an die bekanntesten Fachmänner für Papyrusrestaurierung (Hugo Ibscher) ${ }^{34}$ und Editionen (Ulrich Wilcken) verweist. So sucht er neben der Vermittlung von Ankäufen für Deutsche und deutsche Sammlungen auch die Restaurierung und wissenschaftliche Bearbeitung zu organisieren.

\section{Brief Borchardt, Abusir, an Melchers, Kairo, 19.2.1902}

Handschr. Durchschrift, DAIK Altakten, B XXIXa Korrespondenz Ankäufe „Kleinere deutsche Sammlungen“ 1899-1912.

Abusir 19/2 02

Sehr geehrter Herr Melchers!

Postadresse: Kairo Menahaus

Abdallah kam natürlich sofort nach Eintreffen Ihres werthen Briefes mit den Papyrus an, und habe ich ihm die Kisten N.o 1-3 abgekauft u(nd) auch aus den anderen 4 Kisten noch 6 gute Stücke als Zugabe dazulegen lassen. Gezahlt habe ich ihm dafür $25 £+3 £$ Com(m)ission für seine Bemühungen. Mit der Zahlung bis z(u) Ihrer Rückkehr zu warten, konnte ich dem armen Kerl, dessen Verhältnisse ich ungefähr kenne, nicht zumuthen.

Ich habe die Papyri vorläufig hier $I^{2}$ aufbewahrt $u(n d)$ händige sie Ihnen gern jederzeit aus. Die Ausfuhr hat keine bes(onderen) Schwierigkeiten, die dazu erforderlichen Formalitäten besprechen wir am besten nach Ihrer Rückkehr von Oberägypten.

Für die weitere Behandlung der Papyri würde ich Ihnen empfehlen, dieselben dem Buchbinder Ibscher der Berliner aegyptischen Abtheilung zu übergeben, der sie Ihnen tadellos unter Glas bringt und zusam(m)ensetzt, soweit es noch nöthig ist. Erst dann werden Sie sehen, was für einen guten Kauf Sie gemacht haben. Es sind nach meiner Taxe etwa 40 vollständige Briefe $\mathrm{z}(\mathrm{um}) \mathrm{Th}(\mathrm{eil})$ wohl auch amtlichen Charakters, da ich auf der einen Adresse den Strategen von Apollonopolis als

${ }^{34}$ Hugo Ibscher (*28. September 1874 in Berlin; $† 26$. Mai 1943 ebd.) war der bekannteste Restaurator für Papyri. Zu seiner Tätigkeit vgl. M. Krutzsch: „Geduld will beim Werke sein “. Zur Erinnerung an Hugo Ibschers Aufnahme der selbstständigen Tätigkeit in der Berliner Papyrussammlung 1894, APF 40, 1994, S. 165-166. Seine Bedeutung zeichnet ausführlich sein Sohn nach in: R. Ibscher: Hugo Ibscher zum Gedächtnis, Das Altertum 5, 1959, S. 183-189. 
Absender gelesen habe. ${ }^{3}$ Die wissenschaftliche Bearbeitung macht am schnellsten $\mathrm{u}(\mathrm{nd})$ besten, falls Sie nicht werth darauf legen, einen Bremer Herrn damit zu betrauen, unsere erste Autorität auf dem Gebiete der Papyrusforschung, Prof. Wilcken in Würzburg, dem ich übrigens gern deswegen schreiben will, falls Sie es wünschen.

In der Hoffnung, Sie auf Ihrer Rückkehr aus Oberägypten bald hier bei uns zu sehen, $u(n d)$ mit den besten Empfehlungen an Sie u(nd) Ihre Reisegenossen

Ihr sehr ergebener

Borchardt.

Melchers bedankt sich prompt nach seiner Rückkehr und kündigt für die darauffolgende Woche seinen Besuch an. Die weitere Korrespondenz und die spätere Gegeneinladung nach Bremen sprechen für einen guten Verlauf dieses Besuches.

\section{Brief Melchers, Shepard's Hotel, Kairo, an Borchardt 21.2.1902}

Handschriftliches Original auf Briefkopf der $C^{i e}$ Internationale des grandes Hôtels. DAIK Altakten, B XXIXa Korrespondenz Ankäufe „Kleinere deutsche Sammlungen " 1899-1912.

Sehr geehrter Herr Doctor,

nach ganz interessanter aber heißer Nil-Fahrt nach hier zurück gekehrt, finde ich Ihre freundl(ichen) Zeilen vom 19ten hier vor, womit Sie mir den Ankauf von Papyrus melden und bitte ich Sie meinen herzlichen Dank dafür entgegen nehmen zu wollen.

Zweck dieser Zeilen ist eine Anfrage, ob mein Pflegesohn Herr Grobien $^{35} \&$ ich etwa am nächsten Sonnabend d. 1ten März unsern Besuch $\mathrm{I}^{2}$ machen dürfen? Keine Antwort würde ich vielleicht als Bejahung auffassen dürfen, in welchem Falle wir Morgens 9 Uhr v. hier nach den Pyramiden per Tram fahren würden, um von da zu Esel zu Ihnen zu kommen.

${ }^{35}$ Max Hermann Grobien (*Hongkong 28.12.1871, $\uparrow$ Bremen 12.5.1937) arbeitete als eine Art Privatsekretär für Hermann Melchers. Auch seine vier Brüder verkehrten häufig im Hause Melchers. 
Abgesehen davon, daß es mir von größtem Interesse sein wird, das Feld Ihrer Thätigkeit zu sehen, drängt es mich auch Ihnen persönlich meinen Dank für Ihre Güte auszusprechen und verbleibe ich

Ihr ganz ergebener

Herrmann Melchers

Während seines Besuches bei Borchardt wird auch Melchers' Entscheidung deutlich, keine weiteren Papyri zu erwerben. So schreibt Borchardt am 1. März 1902 an Erman über die deutschen Interessenten an Papyrusankäufen: ${ }^{36}$

4) Bremen, von Herrn Crönert angeregt. Bisher hat aber nur der Vorsitzende der Bremer geographischen Gesellschaft für $28 £$ Papyri (Briefe) dem dortigen Museum geschenkt. Ich habe Zweifel, ob überhaupt Weiteres zu Stande kom(m)t.

Am Rande fügt er dann hinzu: „Nach einer soeben gehabten Unterredung mit Herrn Melchers bin ich sicher, daß nichts Weiteres zu Stande $\operatorname{kom}(\mathrm{m}) \mathrm{t}$.“

\section{Der Gießener Ankauf}

Bereits am selben Tag des Erwerbs der Papyri für Bremen sorgte Borchardt dafür, den gesamten Fund nach Deutschland zu bringen. Dazu greift er eine Bemerkung Kornemanns auf, auch nicht literarische Papyri erwerben zu wollen, und schlägt ihm den Ankauf der noch übrigen Stücke vor.

Brief Borchardt, Kairo, an Kornemann, 19.2.1902

Handschr. Durchschrift, DAIK Altakten, B XII Korrespondenz Ankäufe Giessen 1902-1905, 1907, 1910.

Abusir 19/2 02

Postadresse, Kairo, Menahaus

\footnotetext{
${ }^{36}$ Brief Borchardt, Abusir, an Erman, Steglitz, 1.3.1902. Handschr. Durchschrift, DAIK Altakten, F II Deutsches Papyruskartell. Korrespondenz mit Kgl. Museen zu Berlin 19011906, S. 10.
} 
Sehr geehrter Herr Dr.!

Ihren Brief vom 9. d. M. habe ich erhalten und bin sehr erfreut, daß Sie dem Auftrag zu Papyrusankäufen eine weitere Ausdehnung gegeben haben als ich zunächst dachte. Falls Sie nicht allein litterarische Stücke wünschen, kann ich Ihnen in einiger Zeit - natürlich nicht mit einem Schlage - eine ganz ansehnliche u. instruktive Papyrussam(m)lung für Lehrzwecke zusam(m)enbringen. Daß ich Ihnen keine werthlosen Fragmente erstehen werde, dessen können Sie gewiß sein. Darf ich Sie nunmehr $I^{2}$ bitten, die Ihnen zur Zeit zur Verfügung stehende Summe unter Zurückbehaltung eines kleinen Betrages für die dort zu entrichtenden Transportkosten durch Vermittlung eines beliebigen Bankhauses, das mit dem Kairener Crédit Lyonnais oder der Kairener Banque Ottomane in Verbindung steht, an eine der beiden genannten Banken für mich einzahlen zu lassen. Ich werde es dann von dort im Bedarfsfalle erheben.

Vor einigen Tagen war eine hübsche Briefsam(m)lung - mindestens 60 vollständige Stücke - aus Ešmunejn hier zu haben. Ich habe die bessere Hälfte eben für $28 £$ für das Brem \{ens\}er Museum gekauft, da den Herren der ganze Fund zu theuer war. Es ${ }^{3}$ wäre vielleicht gut auch den Rest für Deutschland zu erwerben und für Sie festzuhalten, falls es noch geht. Ich denke für 15-20 £ ist er zu haben. Die Briefe scheinen mir alle an ein $\mathrm{u}(\mathrm{nd})$ dieselbe Person gerichtet, würden also als Ganzes gewiß interessante Einblicke in das tägliche Leben des römischen Aegyptens ${ }^{37}$ gestatten. Vielleicht sind auch amtliche Briefe darunter, ich habe auf dem einen Bremer Stück einen Strategen von Apollonopolis als Absender gelesen.

Vor gefälschten Ostraka brauchen sie keine große Furcht zu haben. Es läuft einmal eins oder das andere mit unter, das hat aber bei dem Preise der Stücke (10 Pfennig ohne die Transportkosten) nichts zu sagen. Für Straßl'burg habe ich etwa 1000 erworben, für Leipzig werden gerade weitere 1000 gesam(m)melt. Falls sie nach Erledigung des Leipziger Ankaufs auch Ostraka haben wollen, steht dem nichts im Wege.

Mit den ergebensten Grüßen u(nd) vorzüglicher Hochachtung Dr. Borchardt.

Kornemann nimmt den Vorschlag an und bittet Borchardt, die übrigen Papyri aus dem von Bremen erworbenen Fund für ihn zu kaufen. Zu diesem Zweck überweist er 500 Mark nach Kairo, sowie weitere 100

${ }^{37}$ Oder „Aegypters“? 
Mark zum Ankauf von Ostraka. Aus der unten abgedruckten Abrechnung Borchardts geht hervor, daß der Ankauf am 2. Mai stattfand.

\section{Brief Kornemann, Giessen, an Borchardt, Kairo, 7.3.1902}

Handschr. Original, DAIK Altakten, B XII Korrespondenz Ankäufe Giessen 1902-1905, 1907, 1910.

Giessen, Frankfurterstr. $58^{\mathrm{II}}$ 7/III 1902.

Sehr geehrter Herr Doctor!

Wie mir soeben mein Auftraggeber schreibt, sind unter dem 4. d. M. zunächst M. 500 zu Ihren Gunsten an die Bank Crédit Lyonnais in Cairo durch Vermitstlung des hiesigen Bankhauses S. Heichelheim angewiesen worden.

Ich hatte vor einiger Zeit $100 \mathrm{M}$. bei demselben Bankhaus deponiert, die ich in einer Gesellschaft mir befreundeter Herren zum Zweck des Ankaufs von Ostraka gesammelt habe, und hatte das Bankhaus gebeten, bei der ersten Sendung an Sie diese $I^{2} 100$ M. mitgehen zu lassen. Hoffentlich ist das nicht vergessen worden. Ich werde heute oder morgen einmal nachfragen. Ist es nicht geschehen, so werden sie der nächsten Sendung beigegeben werden. Für 100 M. Ostraka möchte ich also auf alle Fälle erworben haben; wenn es wegen des Leipziger Ankaufs noch nicht gleich geht, so doch später.

Nun zu den Papyri. Vor allem besten Dank für Ihren Brief vom 19. Februar. Vorausg[esetzt,] dass noch gute Stücke darun[ter] sind, bin auch ich der Ansi[cht,] dass wir '[den Rest' der Briefsammlung [aus] Ešmunejn] erwerben sollten, schon damit die ganze Sammlung nach Deutschland kommt. Wenn natürlich Wichtigeres und für $\mathrm{I}^{3}$ meine Zwecke noch Empfehlenswerteres auftaucht, bestehe ich nicht auf jener Ansicht. Das Bessere ist des Guten Feind! Ich betone nochmals, der Ankauf für Giessen brauch $<\mathrm{t}>$ nicht mit einem Schlag zu geschehen, sondern je nach dem Angebot 'von [wic]htigen Stücken' ${ }^{38}$ Sind die 600 $M$ verausgabt, so bitte ich um Mitteilung. Sollte plötzlich 'eine' grössere Summe nötig werden, so senden Sie bitte ein Telegramm an mich.

$\mathrm{Da}$ ich zum internationalen Historikerkongress nach Rom gehe, lautet meine Adresse vom 1.-28. April ca. Roma Istituto archeologico Germanico, Via monte Tarpeo (Campidoglio).

${ }^{38}$ Der Zusatz ist links am Rand geschrieben. 
Nach der Versicherung in Ihrem letzten Briefe, dass Sie gewillt sind uns eine instruktive Papyrussammlung für Lehrzwecke zu besorgen, lege ich $I^{4}$ unser Unternehmen mit vollem Vertrauen in Ihre Hände und sage Ihnen schon im voraus für Ihre Bemühungen unseren herzlichen Dank. Gelingt es uns ein pa[ar] Lockvögel hierher zu bekommen, so hoffe ich, noch grössere Summen für diese Zwecke flüssig machen zu können. Zunächst sind im ganzen $2000 \mathrm{M}$. zugesagt.

Mit der Versicherung vorzüglichster Hochachtung und den besten Grüssen

Ihr ergebenster

Dr. Ernst Kornemann

\section{5. Übersendung nach Deutschland und Restaurierung}

Zwar berührt dieser doppelte Ankauf dokumentarischer Papyri nicht unmittelbar die Interessen und Bedenken des preußischen Papyrusunternehmens, doch vereinnahmt Borchardt mit dem Bestreben, über die Vermittlung von Ankäufen hinaus auch die Restaurierung und wissenschaftliche Bearbeitung zu organisieren, weitere Ressourcen Berlins. So stellt er im oben zitierten Brief an Erman vom 1. März 1902 ein Programm für die deutschen Papyruserwerbungen vor, das eine Restaurierung der Ankäufe durch Ibscher vorsieht: ${ }^{39}$

Aber ich bin noch nicht zu Ende. Ich sehe das Heil nicht nur in einem Vermittler der Ankäufe, sondern in einem einigen Reichsbuchbinder ${ }^{40}$ für Papyrus. Ich habe nämlich Herrn Melchers vorgeschlagen, die Papyrus die er für Bremen erworben hat, durch Ibscher unter Glas bringen zu lassen u. sie dann I erst seinem Museum zu schenken. Er hat den Vorschlag acceptiert $\mathrm{u}(\mathrm{nd})$ mich gebeten, bei Ihnen die nöthigen Schritte zu thun, daß Ibscher diese Nebenarbeit erfüllen werde. Falls Sie damit einverstanden sind, sende ich die Papyrus gelegentlich nach Berlin.

Erman weist darauf hin, daß Ibscher in seiner Dienstzeit im Museum Arbeiten für Fremde nur mit besonderer Erlaubnis erledigen kann, es ihm jedoch freistünde, solche Aufträge in seiner Freizeit zu Hause zu erle-

${ }^{39}$ Brief Borchardt, Abusir, an Erman, Steglitz, 1.3.1902. Handschr. Durchschrift, DAIK Altakten, F II Deutsches Papyruskartell. Korrespondenz mit Kgl. Museen zu Berlin 19011906, S. $13 f$.

${ }^{40}$ Wohl eine Anspielung auf Ausdrücke in der Form „Deutschlands Einiger Reichskanzler Fürst von Bismarck“, wie sie in den 1890er Jahren auf Münzen geprägt wurden. 
digen. Indem Borchardt diese Möglichkeit aufgreift, beginnt eine Tradition, in der bei weitem die meisten der von deutschen Sammlungen erworbenen Papyri von Ibscher bearbeitet werden. ${ }^{41}$

\section{Brief Borchardt, Kairo, an Ibscher, Berlin, 7.6.1902}

Handschr. Durchschrift, DAIK Altakten, B XXIXa Korrespondenz Ankäufe „Kleinere deutsche Sammlungen“ 1899-1912.

Cairo 7/6 02

Geehrter Herr Ibscher!

Mit dieser Post sende ich Ihnen ein Packet mit 4 Blechkasten voll Papyrus, welche Herrn Hermann Melchers in Bremen gehören. Derselbe will sie unter Glas bringen lassen u. der dortigen Bibliothek schenken. Ich habe bei Prof. Erman angefragt, ob Sie das Glätten etc. besorgen könnten, und die Antwort erhalten, daß sie dies in Ihrer freien Zeit außerhalb des Museums thun könnten, ohne seiner Erl'²laubnis dazu $\mathrm{zu}$ bedürfen. Während der Dienststunden aber könnten sie fremde Arbeiten natürlich nur nach Maßgabe der vorhandenen dienstlichen Arbeit $\mathrm{u}(\mathrm{nd}) \mathrm{mit}$ besonderer Erlaubnis ausführen.

Da Herrn Melchers wohl nicht damit gedient ist, wenn Sie seine Arbeit nur so nebenher im Museum besorgen, so frage ich hierdurch bei Ihnen an, ob sie diese u. etwa spätere folgende Arbeiten zu Hause ausführen wollen. Ich glaube, daß sie von anderen Sammlungen genug Arbeit bekom(m)en werden, um selbst einen Lehrling zu Hause in Ihrer schwiel ${ }^{3}$ rigen Kunst zu unterrichten u(nd) ihn später arbeiten zu lassen, während Sie im Museum sind. Das einzige Bedenken, was ich habe, ist, daß Ihre Wohnung nicht so feuersicher ist, wie das Museum. Da könnte man aber durch Versicherung der Papyri - natürlich auf Kosten der Besitzer - sich vorsehen.

Wenn Sie diese Arbeit übernehmen wollen, so schreiben Sie mir bitte umgehend. Wenn möglich, würde ich Ihnen dan[n] die andere Hälfte dieser Sammlung von Briefen auch [noch] anschaffen dürfen. Diese $I^{4}$ geht nämlich mit gleicher Post nach Gießen. Sollten Sie die Arbeit nicht übernehmen wollen, so senden Sie das Packet bitte mit ein Paar Zeilen

${ }^{41}$ Vgl. auch die Bemerkung von Ibschers Sohn Rolf aus dem Jahr 1961 (abgedruckt bei H. Essler, Zur Geschichte der Würzburger Papyrussammlung, WJA 33, 2009, S. 176): „Schon zu meines Vaters Lebzeiten befanden sich eine Menge Papyri in unserem Hause in Kleinmachnow, meist solche aus anderen Sammlungen, so etwa Hamburger oder Würzburger Bestände, auch ausländische Objekte." 
an den Besitzer, mit dem Sie auch sich über den Preis einigen mögen, falls Sie die Arbeit ausführen. Für die etwaige Versicherung theile ich Ihnen mit, daß der Kaufpreis der Papyri $28 £=$ rd $50 \mathrm{M}$ betrug.

Mit den ergebensten Grüßen

Dr. Borchardt.

In den vom selben Tag stammenden Briefen, mit denen er den beiden Käufern die Absendung der Papyri mitteilt, weist Borchardt noch einmal auf die Zusammengehörigkeit des Fundes und die Restaurierung durch Ibscher hin und versucht eine gemeinsame Veröffentlichung anzuregen.

\section{Brief Borchardt, Kairo, an Melchers, Bremen, 16.6.1902}

Handschr. Durchschrift, DAIK Altakten, BXXIXa Korrespondenz Ankäufe „Kleinere deutsche Sammlungen“ 1899-1912.

Cairo 16/6 02

Hochgeehrter Herr Melchers!

Von der ägyptischen Abtheilung der Berliner Museen erhielt ich die Antwort, daß der dortige Buchbinder Herr Ibscher Ihre Papyri wegen reichlicher anderer Arbeiten zur Zeit in seinen Dienststunden nicht unter Glas bringen könne, es stehe aber nicht im Wege, daß er sie bei sich zu Hause außerhalb des Dienstes erledige. Ich habe ihm daher die Papyri zugeschickt $u\left(\right.$ nd) ihn ersucht, die Arbeit auszuführen. $\left.\right|^{2}$ Er wird sich jedenfalls mit Ihnen darüber in Verbindung setzen.

Die mir erwachsenden Kosten belaufen sich auf:

7 Piaster für Verpackung

48 Piaster für Zoll, Versicherung, Transport.

3 Piaster Porti.

58 Piaster (oder 12,18 M) zusam(m)en. Um Ihnen Porto zu ersparen, darf ich vielleicht bitten, den Betrag an meinen Bruder Herrn Architekten Heinrich Borchard (sic !), Berlin W7, Bülow Str. 18 II/2 gelegentlich zu senden.

Es wird Sie vielleicht interessieren zu hören, daß ich den $1^{3}$ Rest der von Ihnen erworbenen Briefsammlung noch für die Universität Gießen ankaufen konnte. So ist das Ganze wenigstens für Deutschland gerettet. Gut wäre es, wenn man die ganzen Briefe auch zusammen veröffentlichen könnte. 
Mit den ergebensten Grüßen

Ihr

Dr. Borchardt.

\section{Brief Borchardt, Kairo, an Kornemann, 16.6.1902}

Handschr. Durchschrift, DAIK Altakten, B XII Korrespondenz Ankäufe Giessen 1902-1905, 1907, 1910.

Kairo, 16/6 02

Sehr geehrter Herr Dr.!

Mit letzter Post habe ich Ihnen zwei Packete mit Papyrus zusenden lassen $u(n d)$ sende Ihnen anbei eine Aufstellung über den Stand des Gießener Papyrusfonds. Sie ersehen daraus, daß zur Zeit hier noch 290,0 P (= c. 60 M) zur Verfügung sind.

Die andere Hälfte der Briefsammlung ist, wie Sie wissen, für das Bremer Museum bestimmt und von mir an Herrn $I^{2}$ Buchbinder H. Ibscher, Berlin C 2, Königliche Museen, Aegyptische Abtheilung, zum Glätten abgesandt worden. Ich habe den Herrn gebeten, diese Arbeit in seiner freien Zeit außerhalb des Dienstes auszuführen u(nd) würde Ihnen rathen, sich gleichfalls für Ihre Sachen an ihn zu wenden, da ich annehme, daß Sie in Gießen keine geschulten Arbeiter für solche Arbeiten haben werden.

Mit vorzüglicher Empfehlung

Ihr sehr ergebener Borchardt.

(Blatt 2)

Stand des Gießener Papyrusfonds

am 16ten Juni 1902

21/3 Conto beim Credit Lyonnais

eröffnet $(500 \mathrm{M})$

2370,0 P.

No.

2/5 14 Schachteln Briefe von Machmud

Rifai in Medinet el Fajum

2/5 21 Schachtel Papyri aus Ešmunejn von Abd el Al daselbst

5/6 2 Postkisten für No. 1 u. 2

$1515,5 \mathrm{P}$

16/6 Transport u. Versicherung ders.

Porto der Anzeige

Bestand:

487,5 P

$14,0 \mathrm{P}$

$60,0 \mathrm{P}$

$3,0 \mathrm{P} 2080,0 \mathrm{P}$

$290,0 \mathrm{P}$ 
Aufgestellt

Kairo, den 16ten Juni 1902

Dr. Borchardt.

Beim Ankauf No. 1 handelt es sich um die Papyri aus dem Apolloniosarchiv, zu dem nach P.Giss. II, S. 25 auch die Constitutio Antoniana (P.Giss. 40) gehört. Sie sind also nicht, wie bisher angenommen, von Kornemann selbst auf einer Reise nach Ägypten erworben worden. ${ }^{42}$

Ende des Monats kann Kornemann, der inzwischen nach Tübingen berufen worden war, die Ankunft der Papyri in Giessen bestätigen. Er folgt Borchardts Rat, die Papyri ebenfalls bei Ibscher in Berlin restaurieren zu lassen. Die von ihm kurz erwähnte „Auseinandersetzung mit der Berliner Museumsverwaltung“ ging auf die oben erwähnte Denkschrift der am Museum angesiedelten Preußischen Papyruskommission zurück. Kornemann stellte zusammen mit der Kaiserlichen Bibliothek in Straßburg die Gruppe der Konkurrenten für die von Berlin beabsichtigten Papyruserwerbungen. Daher hatte im Verfolg der Denkschrift der preußische Minister der Auswärtigen Angelegenheiten sich zur Herbeiführung einer Einigung jeweils an die zuständige Regierung, den Kaiserlichen Statthalter in Straßburg und die Großherzoglich Hessische Regierung in Darmstadt, gewandt. ${ }^{43}$ Die weiteren Verhandlungen werden schließlich in den folgenden Monaten zur Gründung des Deutschen Papyruskartells führen.

\section{Postkarte Kornemann, Tübingen, an Borchardt, 27.6.1902 (Post- stempel)}

Handschr. Original, DAIK Altakten, B XII Korrespondenz Ankäufe Giessen 1902-1905, 1907, 1910.

Tübingen, Olgastrasse 7

27/VI.02

${ }^{42}$ Vgl. H.G. Gundel: Papyri Gissenses. Eine Einführung, Giessen 1975 (Kurzberichte aus den Giessener Papyrus-Sammlungen 32), S. 7. In der Aufstellung S. 8 ist der Ankauf No. 2 nicht erwähnt.

${ }^{43}$ Auf Straßburg bezügliche Dokumente sind ediert bei O. Primavesi: Zur Geschichte Des Deutschen Papyruskartells, ZPE 114, 1996, S. 173-187, hier S. 180-187. Der Schritt bei der Großherzoglich Hessischen Regierung wird im Begleitschreiben des preußischen Außenministerium an den Kaiserlichen Statthalter vom 12.3.1902 erwähnt (ebd., Dokument 2, S. 182). 
Sehr geehrter Herr Dr.!

Ich bestätige Ihnen den Eingang der ersten Papyrussendung und der Abrechnung. Die Sendung liegt allerdings noch in Giessen, doch habe ich Schritte gethan, sie hierher weitergehen zu lassen. Ich bin nämlich Ende April als Extraordinarius für alte Gesch. hierher berufen worden. Für die Adresse des Herrn Ibscher, Berlin besten Dank. Ich werde den-

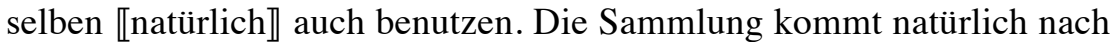
Giessen, daran ändert meine Hierherberufung nichts. Doch bitte ich alle Sendungen von jetzt an meine neue Adresse '(s. oben)' zu richten. Weitere $500 \mathrm{M}$ und 120-140 M für eine Ostraka-Sammlung gehen Ihnen demnächst zu. Über eine interessante Auseinandersetzung mit der Berliner Museumsverwaltung in Sachen meiner Papyrusankäufe schreibe ich Ihnen bald ausführlich. Vorerst herzl. Dank für Ihre Mühewaltung auch im Namen meiner Geldgeber. Mit bestem Gruss

(auf linker Seite weitergeschrieben)

Ihr Ernst Kornemann (nicht $\underline{\underline{H}}$ Hornemann).

Auch die Antwort von Hermann Melchers ist geprägt von Ausdrücken des Dankes. Er lädt Borchardt ein, ihn bei seiner nächsten Rückkehr nach Deutschland in Bremen zu besuchen. Dieser nimmt die Einladung an und legt ihm noch einmal die Bearbeitung der Papyri durch Ulrich Wilcken ans Herz, sowie eine gemeinsame Veröffentlichung zusammen mit dem für Gießen erworbenen Teil. Es ist nicht belegt, ob Borchardt tatsächlich wie angekündigt am Rande des Orientalistenkongresses in Hamburg einen Abstecher nach Bremen gemacht hat, um Melchers zu besuchen. Während dieses Kongresses entwarf er am 8. September 1902 zusammen mit Ulrich Wilcken die Statuten für das künftige Deutsche Papyruskartell. ${ }^{44}$

\section{Brief Melchers, Bremen, an Borchardt, Kairo, 30.6.1902}

Handschr. Original, DAIK Altakten, B XXIXa Korrespondenz Ankäufe „Kleinere deutsche Sammlungen“ 1899-1912.

Bremen, 30. Juni 1902

C. Melchers \& Co.

Telegr.-Adresse: „MELCHERSCO“

\footnotetext{
${ }^{44}$ Das handschriftliche Original im Archiv des DAI Altakten, o. Nr. Deutsches Papyruskartell. Entwurf Satzungen 1902.
} 
Sehr geehrter Herr Dr. Borchardt,

Längst schon war es meine Absicht, Ihnen zu schreiben, und Ihnen nochmals zu danken für die freundliche Aufnahme, welche mein Pflegesohn und ich bei Ihnen gefunden haben. Die Zeit eilt so rasch dahin, daß jene interessanten bei Ihnen verlebten Stunden schon in weite Ferne gerückt schienen, als Ihre freundlichen Zeilen vom 16ten d.M. die gewonnenen Eindrücke lebhaft vor meine Seele zurück riefen.

Mit bestem Dank für Ihre freundl(ichen) Mittheilungen verbinde ich noch mehr meinen erneuten Dank für die gütige Vermittlung des Ankaufs meiner $I^{2}$ Papyri, in Betreff welcher ich mich mit dem Buchbinder Ibscher in Berlin in Verbindung setzen werde.

$\mathrm{Da} ß$ Sie den Rest jener Briefsammlung für die Universität Gießen ankaufen konnten, erfuhr ich mit größtem Interesse, und würde einige Anleitungen Ihrerseits, im Hinblick auf eine zusammenhängende Veröffentlichung der ganzen Briefsammlung, mich zu Dank verpflichten. Ohne Zweifel werden Sie im Laufe des Sommers nach Berlin kommen um 'über' Ihre ausgegrabenen Schätze Bericht zu erstatten, in welchem Falle ich vielleicht keine Fehlbitte thue wenn ich Sie ersuche einen Abstecher nach unserer alten Hansestadt zu machen und mein willkommener Gast zu sein, $\left.\right|^{3}$ bei welcher Gelegenheit wir uns dann über die zu ergreifenden Schritte wegen der in Aussicht zu nehmenden Veröffentlichung unterhalten könnten.

Indem ich mir erlaube Ihrem Herrn Bruder die mir von Ihnen ausgegebenen Unkosten zu überweisen, verbleibe ich in der Hoffnung, Sie in nicht zu ferner Zeit begrüßen zu dürfen, mit freundlichen Grüßen

Ihr sehr ergebener

Hermann Melchers

\section{Brief Borchardt, Kairo, an Melchers, Bremen, 11.7.1902}

Handschr. Durchschrift, DAIK Altakten, B XXIXa Korrespondenz Ankäufe „Kleinere deutsche Sammlungen“ 1899-1912.

Cairo 11/7 02.

Sehr geehrter Herr Melchers!

Ihren freundlichen Brief vom 30ten Juni habe ich erhalten und beeile mich Ihnen die Adresse unseres ersten Papyrusforschers mitzutheilen, den wir in Deutschland und - ohne unbescheidenen Patriotismus - auf der Welt haben. Es ist Prof. Wilcken in Würzburg, Rottendorfer Str. 4. Derselbe wird jedenfalls, wenn er die Veröffentlichung nicht selbst in die Hand nehmen kann, Ihnen einen tüchtigen Gelehrten, der das Papy- 
ruslesen versteht, empfehlen $I^{2}$ können. Er steht übrigens auch mit Herrn Prof. Kornemann in Tübingen in Verbindung, der über die andere Hälfte der von Ihnen erworbenen Briefsammlung zu verfügen hat. Prof. Kornemann, der bisher in Gießen war, ist jetzt nach Tübingen berufen, hat aber die Gießener Papyruserwerbungen bis auf Weiteres noch zu besorgen. Hoffentlich läßt sich eine gemeinsame Veröffentlichung der Bremer $u(n d)$ Gießener Briefe ermöglichen.

Während des Orientalistencongresses werde ich übrigens in Hamburg sein und sehr gern $\left.\right|^{3}$ von da aus einen Abstecher nach Bremen machen, um Ihrer liebenswürdigen Einladung zu folgen. Also auf Wiedersehen im September. Bitte mich Ihrem Herrn Pflegesohn bestens zu empfehlen.

Mit den ergebensten Grüßen

Ihr

Borchardt.

Weit weniger herzlich stellt sich der abschließende Briefwechsel mit Wilhelm Crönert dar. Dieser war seit der Intervention der Preußischen Papyruskommission und dem Eintreffen von Melchers in Ägypten völlig in den Hintergrund geraten. Entgegen seiner Bitte, ihn über die Ereignisse vor Ort zu informieren, schreibt ihm Borchardt erst drei Monate nach Melchers Rückreise, nach Absendung der Papyri, und nachdem er von Crönert einen weiteren Brief mit der Bitte um Nachricht erhalten hatte. Sein Ton ist merklich abgekühlt und nicht frei von Vorwürfen. Insbesondere nimmt er Anstoß daran, daß Crönert in seiner Denkschrift und bei seinem Vortrag in Bremen das ihm von Borchardt mitgeteilte laufende Preußische Papyrusunternehmen nicht erwähnt hatte. Crönert rechtfertigt sich damit, er habe von dem preußischen Vorhaben erst nach der Drucklegung der Denkschrift erfahren und das geheime Vorhaben in Bremen dem Ausschuß mitgeteilt. Die Denkschrift ist auf den 20.12.1901 datiert, als ihm Borchardts Brief vom Vortage sicher noch nicht zugegangen war (vgl. Anm. 16). Daß wenigstens einige Mitglieder der Bremer Kommission wie Reitzenstein und Wilcken vom preußischen Unternehmen wußten, geht aus dem oben zitierten Brief Wilckens vom 29.3.1902 hervor. Freilich stand die auf Öffentlichkeit bedachte Vorgehensweise Crönerts, die nach dem Vorbild der englischen Egypt Exploration Society durch Aufrufe in Denkschriften und öffentlichen Vorträgen private Spender für Papyruserwerbungen gewinnen wollte, in grundsätzlichem Gegensatz zum Unternehmen in Berlin, das gestützt auf die reichen Haushaltsmittel nicht auf private Spender angewiesen war und sich den 
größten Erfolg von Geheimhaltung und raschem Vorgehen versprach. So mußte Crönerts Initiative dem preußischen Vorhaben schon durch ihren Ansatz lästig werden. Die von Borchardt geforderte Bekanntgabe des geheimen Unternehmens in der Denkschrift oder beim Vortrag hätte wohl ebensowenig im Berliner Interesse gelegen. Nach seiner Postkarte vom 1. Juli 1902 kommt Crönert in der Dokumentation für die deutschen Papy-

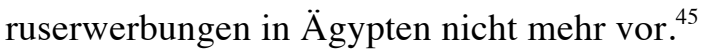

\section{Brief Borchardt, Kairo, an Crönert, Bonn, 19.6.1902}

Handschr. Durchschrift, DAIK Altakten, B XXIXa Korrespondenz Ankäufe „Kleinere deutsche Sammlungen“ 1899-1912.

Cairo 19/6 02

Sehr geehrter Herr Dr.!

Ihren freundlichen Brief vom 12ten d. M. habe ich erhalten. Über die Berliner Ausgrabungen kann ich Ihnen keine Auskunft geben. Fragen Sie bitte bei der Berliner Generalverwaltung direkt an.

Herr Melchers war hier. Ich war sehr verwundert, von ihm zu erfahren, daß Sie meinen Brief vom 19/12 01 in Bremen nicht vorgelegt hatten, wie Sie doch nach Ihrem Brief vom 8/12 01 zu urtheilen, beabsichtigten. Er $\mathrm{I}^{2}$ schien von dem großen preußischen Papyrusunternehmen nicht zu wissen, das Sie ja auch in Ihrer Denkschrift übergehen. Von den Erwerbungen auf seiner Reise wird Ihnen wohl Herr Melchers selbst schon Mittheilung gemacht haben.

In aller Hochachtung

Ihr ganz ergebener

Borchardt.

\section{Postkarte, Crönert, Bonn, an Borchardt, Kairo, 1.7.1902 (Post- stempel)}

Handschr. Original, DAIK Altakten, B XXIXa Korr. Ankäufe Kleinere Sammlungen.

\footnotetext{
${ }^{45}$ Nach seiner Berufung nach Straßburg wohnte er dort ein Mal als Zeuge der Verlosung der Ankäufe bei (18.7.1913) und wirkte im Herbst 1913 bei der Abfassung eines neuen Entwurfs der Kartellstatuten mit.
} 
Bonn, Cassiusgraben 2, den 1 VII 1902

Sehr geehrter Herr Dr,

Herr Melchers hat mir von seinen Erwerbungen noch nichts mitgeteilt. Ich will aber heute einmal bei ihm anfragen. Es wunderte Sie, dass ich, wie es den Anschein hat, mit Absicht von dem Berliner Unternehmen nichts sagte. Darauf erwidere ich, dass ich bei dem Drucke der Denkschrift von jenem Plane noch nichts wusste (erst einige Tage später teilte es mir v. Wilamowitz mit), dass ich aber in Bremen sehr wohl und zwar als Einleitung bei den Verhandlungen des Ausschusses davon redete, mit der Bemerkung, es wäre dringend geboten, dies geheim zu halten. Es drückt mich nicht wenig, dass ein so schöner Plan im Sande zu verlaufen droht. Ich that mein Bestes, mir wars um die Sache, nicht um den Namen zu thun, wie ich denn auch die Ausführung der Grabung nicht übernommen hätte. In aller Hochachtung,

Ihr Crönert.

\section{6. Übergabe der Papyri an die Bremer Bibliothek und Suche nach einem Bearbeiter}

Augenscheinlich erfuhr die heutige Staats- und Universitätsbibliothek (SuUB Bremen) vom Ankauf der später als solche bezeichneten Bremer Papyri erst, als sie ihr als Schenkung angeboten wurden, zumindest verweisen in der SuUB Bremen keine überlieferten Schriftstücke auf Gegenteiliges. Im November 1903 erreichte die damalige „Stadtbibliothek Bremen“, Vorläuferinstitution der SuUB Bremen, ein Schreiben des bremischen Kaufmanns Hermann Melchers, das die Übersendung der Papyri begleitete, die er dem Direktor Heinrich Bulthaupt (1849-1905) für die Bibliothek übergab. Kein Wort wurde jedoch in jenem Übergabeschreiben darüber verloren, auf welchem Wege oder aus welchem Grunde Melchers die Papyri erworben hatte. Der von Borchardt mehrmals betonte Zusammenhang mit dem für Gießen gemachten Ankauf bleibt ebenfalls unerwähnt.

\section{Brief Melchers an Bulthaupt, 28.11.1903}

Staats- und Universitätsbibliothek Bremen, Ordner Eingegangene Briefe an die Stadtbibliothek. 
Bremen, 28. Novbr. 1903.

Herrn Professor Dr. Heinr. Bulthaupt

Stadtbibliothekar

HIER

Sehr geehrter Herr Professor!

Durch Herrn Dr. Ludwig Borchardt, der im Auftrage der deutschen Orient-Gesellschaft zu Berlin, am Fusse der Pyramiden von Abusir Ausgrabungen leitete, erwarb ich im Februar 1902 die beikommenden Papyri. Dieselben stammen aus Eschumên (sic), in Oberägypten, und teilte Herr Dr. Borchardt mir mit, dass es nach seiner Taxe etwa 30 vollständige Briefe seien, zum Teil wohl auch amtlichen Characters da auf einer der Adressen der Name des Strategos von Apollonopolis als Absender genannt sei. Da fast alle Briefe an dieselbe Person gerichtet sind, so werden dieselben in ihrem Zusammenhang ein interessantes Bild des täglichen Lebens jener Zeit enthalten und für den Kulturhistoriker, wie vielleicht auch für den Nationalökonomen wertvoll sein.

Nachdem ich die Briefe in Berlin von sachverständiger Seite zusammensetzen und unter Glas bringen liess, gereicht es mir zum Vergnügen, die Sammlung der Stadtbibliothek zum Geschenk zu überreichen, in der Hoffnung, dass eine wissenschaftliche Bearbeitung der Briefe gelegentlich veranlasst werden kann.

Mit vorzüglicher Hochachtung

Ihr ergebener

Hermann Melchers.

\section{Brief Bulthaupt an Melchers, 30.11.1903}

Staats- und Universitätsbibliothek Bremen, Copir-Buch.

Bremen, d. 30. Novbr. 1903.

Hochverehrter Herr Melchers!

Nehmen Sie meinen verbindlichsten Dank für die wertvolle Sendung, mit der Sie die Stadtbibliothek bereichert und mich insbesondere lebhaft erfreut haben. Sobald ich der Sache näher getreten, werde ich mir gestatten Ihnen Weiteres mitzuteilen, einstweilen drängt es mich nur, mit meinem Dank keinen Tag zu zögern.

Mit ausgezeichneter Hochachtung Ihr ergebenster

gez. Prof. Dr. H. Bulthaupt. 
Offenbar begleitete der Brief Melchers' bereits die ersten Papyri in die Bibliothek. Am 1. Dezember 1903, nur zwei Tage später, schickte Melchers weitere elf Papyri mit dem Hinweis, diese habe er bislang vergessen. Für die übrigen wollte er andere Dispositionen treffen.

\section{Brief Melchers an Bulthaupt, 01.12.1903}

Staats- und Universitätsbibliothek Bremen, Copir-Buch.

Bremen, 1. Decbr. 03

Sehr geehrter Herr Professor.

Für Ihre freundlichsten Zeilen bestens dankend, muss ich um Entschuldigung bitten, einen Theil der Briefe versehentlich hier behalten zu haben, indem dieser Theil schon vor längerer Zeit hier eingetroffen war. - Ich gestatte mir Ihnen die Nr. 9 bis Nr. 19 hiermit zu besendigen, während ich über Nr. 1 - Nr. 8 anderweitig raisonniere.

Freundlichst grüßend

Ihr sehr ergebener

Hermann Melchers

Insgesamt scheint Melchers 90 Papyri erworben zu haben, die alle von Ibscher restauriert, verglast und mit Inventarnummern versehen wurden, bevor Melchers sie erhielt und weiter darüber verfügte. Typisch für Ibschers Arbeitsweise ist der hellgraue Karton, auf dem die Papyri aufgeklebt wurden, sowie der stempelartige Aufdruck der Signatur. Die hier aufgebrachten Signaturen der „Bremer Papyri“ entsprechen den von Melchers bereits benannten Nummern. Die Papyri befinden sich heute noch in den Glasrahmen der damaligen Konservierung.

Von den 90 Inventarnummern wurden 1903 zunächst, wie das Zugangsverzeichnis der Bibliothek belegt, lediglich die Papyri Nr. 20 bis 90 übergeben, dann Nr. 9-19. ${ }^{46}$ Die übrigen scheint Melchers zwischen weiteren öffentlichen Institutionen und Privatleuten aufgeteilt zu haben. So erhielt das heutige Übersee-Museum Bremen drei Stück (P. 3, P. 23, P. 24), das Alte Gymnasium Bremen eines (P. 1, übergeben an die SuUB Bremen 1973) und das heutigen Focke-Museum - Bremer Landesmuseum

${ }^{46}$ Die Eintragung im Zugangsbuch stammt vom 5.12.1903, vgl. T. Elsmann, Die ,Bremer Papyri` - Zur Geschichte einer Sammlung, in: T. Schmitt, M. Böhme (Hg.): Welt aus Schnipseln. Papyrus-Texte aus dem Alten Ägypten, Bremen 2007, S. 53-56, hier S. 55. 
für Kunst und Kulturgeschichte zwei (P. 7 und P. 8, übergeben an die SuUB Bremen 1965). Ein weiterer Papyrus scheint an G. Bergfeld gegangen zu sein. Er ist heute verschollen, wurde jedoch 1915 als SB I 4630 ediert und weist denselben Absender und Adressaten wie P.Brem. 56 auf. ${ }^{47}$ Vermutlich gehört er zur Gruppe der vier Inventarnummern, deren Verbleib bis heute ungeklärt ist (P. 2 und P. $4-6){ }^{48}$ Die Übergabe der Papyri an das heutige Übersee-Museum erfolgte einige Monate früher als das Anschreiben an die Bibliothek. In einem Schreiben vom 25. Juli 1903 bat Melchers Hugo Schauinsland, den Direktor des Museums, die Papyri bei ihm im Contor ,zu besichtigen und einen Theil derselben für Ihr Museum in Empfang zu nehmen." ${ }^{* 49}$

Bemerkenswert ist, dass der größte Teil der von Melchers erworbenen Papyri an die wissenschaftliche Bibliothek ging; war doch zuvor stets von der Erwerbung von Papyri „für das Museum“ die Rede. Dabei teilte der Kaufmann den betreffenden Einrichtungen nicht mit, dass er noch Papyri an andere Institutionen in Bremen verschenkt habe. Ihre Aufteilung zeigt, dass Melchers die „Bremer Papyri“ nicht als vollständige, in sich abgeschlossene Sammlung wahrnahm.

In der wissenschaftlichen Bibliothek Bremens erhielten die Papyri neben den Inventarnummern Ibschers keine neue, bibliotheksspezifische Verzeichnung. Sie werden damit noch heute als eigenständige Sammlung innerhalb der Bestände der Staats- und Universitätsbibliothek Bremen geführt. Verzeichnet sind sie nach wie vor im Zugangsverzeichnis sowie im Standortkatalog der Handschriften und Manuskripte; in beiden Fällen als „Papyri Melchers“, die Bezeichnung „Die Bremer Papyri“, unter der sie heute noch bekannt sind, etablierte sich erst mit Wilckens Publika-

${ }^{47}$ Die Ausgabe erfolgte direkt im Sammelbuch nach einer dem Herausgeber F. Preisigke privat von Schubart zur Verfügung gestellten Abschrift. Beim dort genannten Besitzer handelt es sich wohl um Gottfried Bergfeld von der Bremer Silberwarenfabrik Koch \& Bergfeld, vgl. T. Elsmann, Die ,Bremer Papyri - Zur Geschichte einer Sammlung, in: T. Schmitt, M. Böhme (Hg.): Welt aus Schnipseln. Papyrus-Texte aus dem Alten Ägypten, Bremen 2007, S. 53-56, hier S. 55.

${ }^{48}$ „Bereits Wilcken nahm an, dass Hermann Melchers damit Freunde und Bekannte bedacht haben könnte.“ (T. Elsmann, Die ,Bremer Papyri“ - Zur Geschichte einer Sammlung, in: T. Schmitt, M. Böhme (Hg.): Welt aus Schnipseln. Papyrus-Texte aus dem Alten Ägypten, Bremen 2007, S. 53-56, hier S. 55).

${ }^{49}$ Brief von Hermann Melchers an Hugo Schauinsland, Bremen, 25.07.1903, in: B. von Briskorn, Zur Sammlungsgeschichte afrikanischer Ethnographica im Übersee-Museum 1841-1945, Gelsenkirchen u.a. 2000, S. 264. Vgl. den unten abgedruckten Brief Ulrich Wilckens an Henry Seedorf vom 15.05.1914. 
tionsband. Seit 2013 sind die Papyri digitalisiert und online frei zugänglich. ${ }^{50}$

Nachdem die Papyri in der wissenschaftlichen Bibliothek Bremens angelangt waren, stellte sich die Frage nach der Bearbeitung. Melchers hatte zwar in seinem Begleitschreiben bei Übersendung der Papyri die Hoffnung ausgesprochen, ,dass eine wissenschaftliche Bearbeitung der Briefe gelegentlich veranlasst werden kann“",51 jedoch Borchardts mehrmals wiederholten Vorschlag, Wilcken mit der Veröffentlichung zu betrauen, nicht weitergegeben. Die Bibliothek selbst hatte zu jenem Zeitpunkt nur einen fest angestellten wissenschaftlichen Mitarbeiter, den Direktor Heinrich Bulthaupt (1849-1905, Leiter der Bibliothek seit 1879), der Unterstützung bei seinen wissenschaftlichen Aufgaben vom Bürovorsteher Hubert Wania bekam. Im Zugangsverzeichnis der Bibliothek wurden somit lediglich die Angaben über die Papyri zusammengefasst, die bereits im Schreiben von Melchers nach den Angaben Borchardts erwähnt worden waren.

Die „wissenschaftliche Bearbeitung der Briefe“ musste damit extern geschehen. Die damalige Stadtbibliothek bemühte sich anscheinend sogleich um einen Bearbeiter, hatte jedoch keinen Erfolg damit, junge Philologen für die Edition der Papyri zu gewinnen. Im April 1904 schrieb Bibliotheksdirektor Bulthaupt an Senator Dr. Rudolf Ehmck, ${ }^{52}$ den Vorsitzenden der Deputation für die Stadtbibliothek, mit der Bitte, ihm eine Summe von 250 Mark für die Übersetzung der Bremer Papyri zur Verfügung zu stellen, um damit einen geeigneten Gelehrten ,anlocken“ zu können. Antrag, Verhandlung und Mitteilung über die Bewilligung haben sich in verschiedener Form erhalten.

\section{Brief Bulthaupts an Ehmck 23.04.1904}

Staats- und Universitätsbibliothek Bremen, Copir-Buch.

Bremen, 23. April 1904.

Herrn Senator Dr. Ehmck

Vorsitzer der Deputation für die Stadtbibliothek.

${ }^{50}$ Siehe <http://nbn-resolving.de/urn:nbn:de:gbv:46:1-914>.

${ }^{51}$ Dokument 1: Schreiben Hermann Melchers an Heinrich Bulthaupt, 28.11.1903, in: Staats- und Universitätsbibliothek Bremen, Ordner Eingegangene Briefe an die Stadtbibliothek.

${ }^{52}$ Dietrich Rudolf Ehmck (*Bremen 13.1.1836, † ebd. 17.3.1908) war 1863 Senatssekretär, 1875 Senator geworden. 


\section{Hochgeehrter Herr Senator!}

[...]

Gestatten Sie mir gleichzeitig Ihnen eine Bitte vortragen zu dürfen. Die Melchersche Sammlung von Papyri, derer sich die Stadtbibliothek erfreut, ist nun bereits zum zweiten Male unter den Händen eines jungen Philologen gewesen, der sich mit ihrer Übersetzung beschäftigte; keiner der jungen Herren hat aber bei der Arbeit verdauert, und es fehlt mir an Mitteln dazu anzulocken. Das hoffe ich aber im Stand zu sein, wenn ich dem Übersetzer Aussicht auf eine neuere Belohnung machen könnte. Vielleicht hätten Sie die Güte, vielleicht aus dem Senatfonds, den Betrag von, sagen wir 250 Mark auszuwerfen, mit dem die Übersetzung für einen jüngeren Gelehrten meines Erachtens anständig bezahlt sein würde? Ich könnte mich dann sofort nach einer geeigneten Kraft umthun.

Hochachtungsvoll und ergebenst

Heinr. Bulthaupt.

Ein entsprechender Betrag von 250 Mark zur wissenschaftlichen Erforschung der Papyri wurde nach einer Sitzung des Senats der Freien Hansestadt Bremen am 17. Mai 1904 bewilligt. $^{53}$

\section{Brief Ehmcks an Bulthaupt, 18.05.1904}

Staats- und Universitätsbibliothek Bremen, Ordner eingegangene Briefe.

VII.

Bremen, den 18. Mai 1904

Auf Ihren Bericht vom 23. vor. Mts. hat der Senat sich bereit erklärt, für die Herstellung einer Abschrift und Uebersetzung der durch Herrn Hermann Melchers der Stadtbibliothek geschenkten Papyrus-Sammlung eine Vergütung bis zum Belauf von $250 \mathrm{M}$ zur Verfügung zu stellen, sobald ein dafür vorgesehener geeigneter Gelehrter sich zur Ausführung der Arbeit in verbindlicher Weise verpflichtet hat. Der festzusetzende Betrag würde nach Vollendung der Arbeit auszuzahlen sein. Ich ersuche Sie hiernach, zur Ermittlung einer geeigneten und zur Uebernahme der Arbeit bereiten Kraft sich bemühen und mir sodann weiter berichten

${ }^{53} \mathrm{Vgl}$. Akte, betr. Übersetzung des griechischen Textes von 30 der Stadtbibliothek von Herm. Melchers überwiesenen Papyri. Staatsarchiv Bremen, S. 19. Nr. 25 Senatsregistratur. Auszug aus den Senatsprotokollen vom 17. Mai 1904. Seite 397. 
zu wollen, damit ich einen entsprechenden definitiven Beschluss des Senats herbeiführen kann.

Der Vorsitzer

Der Deputation für die Stadtbibliothek.

Ehmck

Herrn

Stadtbibliothekar Professor Dr. Bulthaupt

Hier,

IN. 2700 A.

Mit der bewilligten Summe versuchte Bulthaupt erneut erfolglos, einen Philologen in Bremen für die Bearbeitung zu gewinnen.

\section{Brief Bulthaupt an Hrn. Lillges, 27.05.1904}

Staats- und Universitätsbibliothek Bremen, Copir-Buch.

Bremen, 27. Mai 04.

Herrn Dr. Fr. Lillges

Hier.

Geehrter Herr Doctor!

Für Bearbeitung der der Stadtbibliothek von Herrn Melchers geschenkten 30 Papyri hat der Senat einen Betrag von M. 250- zur Verfügung gestellt. Darf ich mir daraufhin die freundliche Anfrage erlauben, ob Sie vielleicht geneigt wären, die Arbeit zu übernehmen?

Mit freundl. Gruß

Hubert Wania

i.A.

\section{Bearbeitung durch Ulrich Wilcken}

Nach diesem vergeblichen Versuch, einen ortsansässigen Bearbeiter zu finden, wandte Bulthaupt sich schließlich an den bekanntesten deutschen Fachvertreter, Ulrich Wilcken, der zur Zeit in Halle lehrte, mit der Bitte, einen geeigneten jungen Gelehrten vorzuschlagen. 


\section{Brief Bulthaupt an Wilcken, 09.06.1904}

Staats- und Universitätsbibliothek Bremen, Copir-Buch.

Bremen, 9. Juni 1904.

Herrn Prof. Dr. U. Wilcken

in Halle.

Sehr geehrter Herr!

Ich gestatte mir die ergebene Anfrage an Sie zu richten, ob Sie mir einen jüngeren Gelehrten denn empfehlen könnten, der geneigt wäre die Papyri die die hiesige Stadtbibliothek vor einiger Zeit schenkweise erhalten hat zu übersetzen. Der hohe Senat hat für diese Arbeit einen Betrag von M. 250 - zur Verfügung gestellt, der meines Erachtens als ein angemessenes dafür, da die meisten Blätter nur von kleinem Umfange sind, bezeichnet werden darf. Notwendig würde es allerdings ein, daß der Betreffende sich hierher beehrt, da die unter Glas befindlichen Stücke nicht gut versendbar sind.

Hochachtungsvoll

Heinr. Bulthaupt

Stadtbibliothekar.

Wilckens Antwort ist nicht erhalten, doch wird dem Fachmann nicht zweifelhaft gewesen sein, daß die „Herstellung einer Abschrift und Uebersetzung" vollwertige Editionen voraussetzte und damit umfangreichere Vorkenntnisse und weit mehr Zeit erforderte, als Bulthaupt angenommen hatte. Wilcken scheint die Arbeit sogleich selbst übernommen und konkrete Vorschläge zur Übersendung der Papyri an die Universitätsbibliothek in Halle gemacht zu haben. Bulthaupt greift alle Vorschläge gerne auf, ist aber wohl weiterhin im Unklaren über die Einzelheiten der papyrologischen Arbeit. Er zielt auf eine Übersetzung für ein allgemeines Publikum. Diese ,soll den Besuchern der Stadtbibliothek, also Gelehrten wie Laien zugängig gemacht werden, immerhin wäre es erwünscht, wenn der Text vorab in das übliche Schrift-Griechisch übertragen werden könnte. ${ }^{654}$ Wilckens Beschäftigung mit den Bremer Papyri sollte sich von 1905 bis zum Erscheinen des Publikationsbandes 1935 erstrecken.

${ }^{54}$ Brief Bulthaupt an Wilcken, 15.06.1904, Staats- und Universitätsbibliothek Bremen, Copir-Buch. Hervorhebung von M. Hermes. 


\section{Brief Bulthaupt, Bremen, an Wilcken, Halle, 15.06.1904}

Staats- und Universitätsbibliothek Bremen, Copir-Buch.

Bremen, d. 15. Juni 1904.

Herrn Professor Dr. U. Wilcken in Halle.

Hochverehrter Herr Professor!

Mit meinem verbindlichsten Dank für das gütige Interesse, das Sie der Uebersetzung der bremischen Papyri schenken, verbinde ich den Ausdruck der Freude darüber, dass Sie persönlich sich hiermit bemühen wollen. Natürlich folge ich den gütigst erteilten Rathschlägen, lasse die Sammlung der Kgl. Universitätsbibliothek zugehen und bitte Sie in Allem nach Ihren Vorschlägen zu verfahren, natürlich auch betreffs der Veröffentlichung in Ihrem Archiv. Für uns eilt die Sache durchaus nicht. Die Uebersetzung soll den Besuchern der Stadtbibliothek, also Gelehrten wie Laien zugängig gemacht werden, immerhin wäre es erwünscht, wenn der Text vorab in das übliche Schrift-Griechisch übertragen werden könnte.

Hochachtungsvoll

Prof. Dr. H. Bulthaupt,

Stadtbibliothekar.

Auf Wilckens Zusage hin geht Bulthaupt von seiner Bedingung ab, daß die Bearbeitung der Stücke in Bremen erfolgen solle, und erklärt sich mit der Versendung auf dem Postwege einverstanden. Nach einer ersten Anfrage noch im Juni 1904 werden schließlich ein dreiviertel Jahr nach Wilckens positiver Antwort auf die Anfrage der Bremer Stadtbibliothek im März 1905 die Glasplatten an ihn versandt. ${ }^{55}$ Die „30 Briefe“ von denen Wania in seinem Schreiben spricht, beziehen sich möglicherweise auf die von Melchers erwähnten 30 vollständigen Briefe, aus denen die Sammlung bestand, möglicherweise jedoch auch auf die 30 Glasplatten, in denen die Papyri aufbewahrt wurden. Sehr sensibel reagierte man in der

${ }^{55}$ Die erste Anfrage ist der Brief Hubert Wanias (Bürovorsteher Stadtbibliothek Bremen) an Wilcken, 24.06.1904, Staats- und Universitätsbibliothek Bremen, Copir-Buch: „Bremen, 24. Juni 04.

Hochgeehrter Herr!

In Vertretung von Herrn Professor Bulthaupt, der Krankheitshalber einen dreimonatigen Urlaub angetreten hat, gestatte ich mir die ergebene Anfrage, ob Ihnen die Übersendung der Papyri schon jetzt erwünscht wäre?

Hochachtungsvoll, Hubert Wania“" 
Bibliothek auf Beschädigungen dieser Glasplatten beim Transport. So auch, als Wilckens Meldung eintrifft, daß neben der bereits bei Absendung beschädigten Platte noch vier weitere gesprungen waren.

\section{Brief Wania an Wilcken, 22.03.1905}

Staats- und Universitätsbibliothek Bremen, Copir-Buch.

Bremen, 22. März 05.

Herrn Professor Dr. U. Wilcken in Halle a.S.

Sehr geehrter Herr!

Ich beehre mich Ihnen ergebenst anzuzeigen, daß die 30 Papyri heute in drei Paketen an die bezeichnete Adresse abgingen und bitte, ihre unversehrte Ankunft umgehend anzeigen zu wollen.

Das Schutzglas von einem war schon bei der Absendung lädiert.

Hochachtungsvoll

Für Prof. Dr. Bulthaupt

Wania i.V.

\section{Brief Wania an Wilcken, 24.03.1905,}

Staats- und Universitätsbibliothek Bremen, Copir-Buch.

Bremen, 24. März 05

Herrn Prof. Dr. U. Wilcken

in Halle a $S$.

Sehr geehrter Herr!

Im Auftrage von Herrn Professor Bulthaupt, der heute zuerst während seiner noch nicht gehobenen Erkrankung wieder in der Bibliothek erscheinen konnte, möchte ich Ihnen freundlich danken für Ihr letztes gefl. Schreiben, und seiner Freude über Ihre Mitteilung Ausdruck geben, daß die Papyri sich von besonderem Werte erweisen könnten. Daß sie, die wir schon so schön und sicher glaubten verpackt zu haben, nun doch nicht ganz unbeschadet übergekommen sind, hat uns allerdings unangenehm überrascht.

Hochachtungsvoll

Hubert Wania. 
Als Wilcken nach Leipzig umzog und die Papyri dorthin überführen wollte, spielte die Frage der Verpackung und der Versicherung als Konsequenz der Beschädigungen beim Versand von Bremen nach Halle eine bedeutende Rolle. So legt Henry Seedorf (1863-1922, Stadtbibliothekar von 1906 bis zu seinem Tode), Wert auf eine hohe Versicherungssumme und die Aufbewahrung in der dortigen Bibliothek.

\section{Brief Seedorf an Wilcken, 16.03.1906}

Staats- und Universitätsbibliothek Bremen, Copir-Buch.

Bremen, den 16. März 1906.

Herrn Professor Dr. U. Wilcken in Halle a.S.

Hochgeehrter Herr Professor!

Gern erteile ich Ihnen die Erlaubnis, die in Ihrem Besitz befindlichen Papyri der Bremer Stadtbibliothek zum Zweck ihrer weiteren wissenschaftlichen Erforschung nach Leipzig zu überführen. Ich bitte nur, sie beim Transport auf Kosten der hiesigen Stadtbibliothek mit einer höheren Summe, etwa 5000 Mark, versichern zu wollen. Am liebsten wäre es mir, wenn die Papyri in Leipzig innerhalb der Kgl. Bibliothek aufbewahrt werden könnten, da ich vermute, daß die Feuersgefahr im Bornerianum größer ist.
Mit vorzüglicher Hochachtung
Ihr ergebenster
Prof. H. Seedorf
Stadtbibliothekar.

Die erste veröffentlichte Erwähnung von Wilckens Arbeit an den Bremer Papyri stammt aus seiner Leipziger Zeit; sie findet sich in der Miszelle ,"Е $\pi \tau \alpha \kappa \omega \mu$ í $\alpha$, eine neue Papyrusquelle“ (APF 4, 1908, S. 163f.) ${ }^{56} \mathrm{Im}$ Jahr 1914 war dann die Erstellung des Inventars mit Verfasserangaben und Inhaltstiteln abgeschlossen und Wilcken sandte die Originale nach Bremen zurück. In seinem Begleitschreiben an die Bibliothek geht er vor allem auf die Beschädigungen an den Glasrahmen ein und bietet an, die Kosten für die Versicherung zu übernehmen.

\footnotetext{
${ }^{56}$ Weitere Erwähnungen der Sammlung mit Zitaten aus einzelnen Texten folgen in den nächsten Jahren, etwa APF 4, 1908, S. 164, S. 376 Anm. 1, S. 385-388, APF 5, 1913, S. 245-247. ASGW 27, 1909, S. 797.
} 


\section{Brief Wilcken an Seedorf, 15.05.1914}

Staats- und Universitätsbibliothek Bremen, msc 0152. ${ }^{57}$

Freitag d. 15.5.14

Hoch geehrter Herr Professor!

Hierdurch teile ich Ihnen mit, daß heute Mittag Ihre Papyri (30 Platten) wohl verpackt der Post übergeben sind. Ich habe mir von einem hiesigen Glasgeschäft den besten Packer geben lassen, und dieser hat in meiner Gegenwart heute Vormittag die Verpackung auf's sorgfältigste durchgeführt, sodaß ich hoffe, daß die Papyri wohlbehalten bei Ihnen eintreffen. Wir haben sie in 4 Kisten verpackt, von denen ich $3 \mathrm{zu}$ je 1000 Mark, die 4te, die größte, mit 2000 Mark versichert habe. Für Ihr freundliches Anerbieten, die Kosten zu übernehmen, danke ich Ihnen sehr, $\left.\right|^{2}$ bitte aber, mir zu gestatten, daß ich sie selber trage.

Uebersandt waren mir im März 190530 Platten, die Sie jetzt zurück erhalten. Die Nummern der Papyri laufen bis 90, aber die Nr. 1-8 und 23-24 sind nicht zugeschickt worden. Es scheint, daß Herr Melchers diese anderweitig verschenkt hat.

Bei dem Transport von Bremen nach Halle waren, wie ich nach Empfang der Sendung am 25.3.05 Ihrer Verwaltung angezeigt, abgesehen von einem Stück, dessen Platten bereits bei der Absendung in Bremen lädiert waren (laut Brief Ihrer Verwaltung vom 22.3.05), noch 4 weitere Stücke lädiert worden, $\beta^{3}$ insofern bei 2 Stücken die Glasplatten einseitig, bei 2 beiderseitig gesprungen waren. Wie ich schon damals in jenem Brief hervorhob hatten die Papyri selbst dadurch in keiner Weise gelitten, sie waren unversehrt angekommen, wie ich sie Ihnen auch unversehrt zurückschicke. Trotzdem habe ich es für gut gehalten die beiden Stücke, deren Gläser auf beiden Seiten sehr stark gesprungen waren, in Leipzig neu verglasen zu lassen, da sonst eventuell doch ein Schaden hätte eintreten können. Das sind die beiden Nummern 12 und 47-50, die Sie daher mit weißem Papier umschlossen finden werden. Ich bemerke dazu, daß derartige Papierverschlüsse, wie sie in manchen Sammlungen $I^{4}$ üblich sind, im Interesse der Conservierung der Papyri durchaus genügen.

$\mathrm{Zu}$ den anderen 3 Papyri, die ich, wie gesagt, gleichfalls von vornherein mit gesprungenen Glasplatten erhalten hatte, ist im Laufe der Jahre nur noch ein Sprung bei der Nr. 10 hinzugekommen. Die 4 Stücke, die hienach also gesprungene Platten haben, sind:

Nr. 10, 25-28, 60-65, $\underline{89}$.

${ }^{57}$ Abbildungen sind verfügbar unter <http://nbn-resolving.de/urn:nbn:de:gbv:46:1-971>. 
Ich habe bei diesen Stücken aber draufgeschrieben: „Vorsicht, Glas gesprungen“, damit Sie sie beim Auspacken besonders vorsichtig herausnehmen können. Im Uebrigen ist, wie ich wiederhole, durch einen solchen Sprung eine Gefahr für die Papyri selbst nicht gegeben. $I^{5}$

Meinen Bericht über Ihre Papyri hoffe ich bis morgen Abend beenden zu können, sodaß er am Montag gleichzeitig mit den Originalen bei Ihnen eintrifft. Sollte ich aber auch den Sonntag noch dazu nötig haben, so werden Sie Dienstag, spätestens Mittwoch in den Besitz des Berichtes kommen. Es macht mir viel Vergnügen, teils durch Transcription, teils durch Uebersetzungen oder Paraphrasen Ihnen einen Einblick in den Inhalt Ihrer wertvollen Sammlung zu geben. Ich habe $I^{6}$ diese Arbeit um so lieber gemacht, als sie ja die einzige Form ist, in der ich Ihnen meine Dankbarkeit ausdrücken kann. Wenn ich, wie ich hoffe, im nächsten Heft des Papyrusarchivs mit der Publication beginne, werde ich wahrscheinlich einzelne Stellen nochmals vorher am Original prüfen wollen. Vielleicht wird es mir dann möglich sein, zu diesem Zweck nach Bremen zu kommen. Sonst würde ich Sie an Ihr freundliches Versprechen erinnern, $I^{7}$ mir eventuell einzelne Nummern nochmals auf kurze Zeit zur letzten Revision zuzusenden.

In vorzüglicher Hochachtung

Ihr ergebener

UWilcken

Der von Wilcken angekündigte Bericht über die Sammlung mit Transkriptionen, Übersetzungen und Paraphrasen der Stücke ist auf den folgenden Tag (16.5.1914) datiert. ${ }^{58}$ Bald nach Übersendung der Daten und Papyri an die Stadtbibliothek nimmt der damalige Bibliotheksdirektor Seedorf die Gelegenheit wahr, dem Spender Hermann Melchers noch einmal zu danken für den ,kostbaren Schatz [...], der wesentlich dazu beitragen wird, das Ansehen des Instituts zu erhöhen.“ Und kurze Zeit darauf konnten einige der Papyri im Rahmen des Neuphilologentages erstmals ausgestellt werden. Sogar den Bremer Nachrichten war diese Ausstellung eine Ankündigung wert, in der auch einige für den allgemein interessierten Besucher besonders interessante Stücke benannt wurden: „Zum ersten Male sollen dabei die wertvollen Papyri gezeigt werden, die Herr Hermann Melchers der Bibliothek geschenkt hat. Einer der hervorragendsten Papyrusforscher, Herr Professor Dr. Ulrich Wilcken in Bonn, hat sie untersucht und mit einem Kommentar versehen. Sie stammen aus der

${ }^{58}$ Staats- und Universitätsbibliothek Bremen, msc 0152 (urn:nbn:de:gbv:46:1-971), S. 15-17. 
oberägyptischen Stadt Heptakomia und beziehen sich zum größten Teil auf einen Apollonios, der um 115 n.Chr. der oberste Beamte in jener Gegend war. Besonderes Interesse haben für das größere Publikum Volkszählungs-Deklarationen einzelner Bürger, Zahlungsanweisungen auf Banken, eine Urkunde über ein hypothekarisch sichergestelltes Darlehen und ein Privatbrief einer Dame, worin erwähnt wird, daß in der Stadt eine Straßendemonstration zur Erlangung höherer Löhne stattgefunden hat.“" ${ }^{* 59}$

\section{Brief Seedorf an Melchers, 28. 5.1914}

Staats- und Universitätsbibliothek Bremen, Copir-Buch.

Bremen, den 28. Mai 1914.

Herrn Hermann Melchers.

Hier

Sehr geehrter Herr Melchers!

$\mathrm{Zu}$ meiner Freude kann ich Ihnen mitteilen, daß die von Ihnen der Stadtbibliothek im Jahre 1903 geschenkten Papyri uns vor kurzem von Herrn Professor Dr. Ulrich Wilcken in Bonn, dem sie zur Untersuchung gesandt waren, mit einem aufschlußreichen Kommentar wieder zurückgestellt sind. Herr Prof. Wilcken hat fast alle Stücke genau bestimmt, ihren Inhalt näher gekennzeichnet und bei den meisten Umschriften in die übliche griechische Schrift mit Übersetzungen gegeben. Ich gestatte mir, diesem Schreiben einen kurzen Auszug aus seinem Kommentar $l^{2}$ beizufügen, der ein Bild von der Mannigfaltigkeit des Inhalts der Papyri gibt, und bitte Sie, dies Exemplar des Auszugs als Ihr Privateigentum zu betrachten, jedoch keine Veröffentlichung daraus zu gestatten. Jetzt erst ist es möglich, den Wert Ihrer Schenkung genau zu ermessen, und so benutze ich gern diesen Anlaß, um Ihnen im Namen der Stadtbibliothek nochmals den verbindlichsten Dank auszusprechen. Sie haben die Stadtbibliothek damit um einen kostbaren Schatz bereichert, der wesentlich dazu beitragen wird, das Ansehen des Instituts zu erhöhen.

In vorzüglicher Hochachtung

Ergebenst

Der Stadtbibliothekar

H. Seedorf.

${ }^{59}$ Vgl. Zeitungsanzeige in den Bremer Nachrichten, 01.06.1914. 
Im Januar 1915 erhielt Wilcken die drei an das heutige Überseemuseum gegangenen Papyri nach Bonn übersandt und schickte sie im September mit Inhaltsangaben zurück, ${ }^{60}$ doch konnte er den eigentlichen Editionsband erst zwanzig Jahre später abschließen. Darin wurde neben den damals in der Bibliothek und im Überseemuseum befindlichen Papyri auch ein von Melchers an das Alte Gymnasium gegebener Papyrus (P.Brem. 1) mit berücksichtigt.

Ein Brief Wilckens an Georg Steindorff aus dieser Zeit belegt eindrücklich, wie viel eingehende Forschungsarbeit die Editionen erforderten. Seine Frage entspringt dem Zweifel an der Angabe in einem Urlaubsgesuch des Architekten Herodes an Apollonios in P.Brem. 15, Z. 30f., wonach die Schiffahrt vom Hermopolites nach Alexandrien in zwei Tagen zu bewerkstelligen wäre. ${ }^{61}$

\section{Brief Wilcken, Berlin, an Steindorff, Leipzig, 12.11.1935}

Handschriftl. Original, ÄMULA NL Georg Steindorff, Korrespondenz.

\section{Liebes Bengelchen!}

Ich möchte Dir eine Frage vorlegen, die mich bei den Bremer Papyri, die ich jetzt herausgeben will, beschäftigt. Da will Einer zu Schiff von Hermopolis aus - wohl von einem nördlichen Hafen des Gaus aus nach Alexandrien fahren und sagt: „Zwei Tage beträgt die Entfernung.“ Ist das möglich, diese Fahrt in 2 Tagen zu machen? Freilich ist zu bedenken, daß er das am 29. August schreibt, also zur Zeit der stärksten Nilschwelle, wo der Strom wohl reißend schnell geht. Die Schriftstellerangaben (wie Diod. 3,34: 10 Tage v. Alex. nach Aethiopien etc.) reden immer von der Stromauffahrt. Das ist etwas ganz anderes. Aber 2 Tage kommt mir doch verflucht kurz vor. Freilich sagt er auch nicht, daß er es gemacht hat, sondern er behauptet diese kurze Distanz in einem Urlaubsgesuch. Immerhin muß er seinem Herrn, dem Strategen,

${ }^{60}$ P.Brem. S. 7; vgl. T. Elsmann, Die ,Bremer Papyri ${ }^{\star}$ - Zur Geschichte einer Sammlung, in: T. Schmitt, M. Böhme (Hg.): Welt aus Schnipseln. Papyrus-Texte aus dem Alten Ägypten, Bremen 2007, S. 53-56, hier S. 55f.

${ }^{61}$ Im Kommentar zur Stelle (P.Brem., S. 50) zitiert Wilcken dann Steindorffs Antwort, nach der die zwei Tage bei reißender Nilschwemme stromabwärts möglich erscheinen. 
glaubwürdig sein. Hältst Du es für möglich? Ich wäre Dir sehr dankbar, wenn Du mir recht bald ein Kärtchen darüber schreiben wolltest.

Wie würde ich mich freuen, Dich mal wiederzusehen! Es war das letzte Mal so schön! Ich wünsche und hoffe, daß es Dir und den Deinen gut geht, und bleibe in alter Freundschaft Dein

Ulrich W.

Nach dem Erscheinen von Wilckens Editionsband im Jahr $1936^{62}$ wurden 1966 von Herwig Maehler noch zwei weitere Papyri, die in das Focke-Museum gelangt waren, veröffentlicht. ${ }^{63}$ Von drei der insgesamt 90 von Ibscher verglasten Papyri fehlt weiterhin jede Spur.

${ }^{62}$ U. Wilcken, Die Bremer Papyri, Berlin 1936.

${ }^{63}$ SB X 10277 und 10278. H. Maehler, Zwei neue Bremer Papyri, CdÉ 41, 1966, S. 342-353. 